\title{
Spectroscopy across the brown dwarf/planetary mass boundary
}

\section{Near-infrared JHK spectra ${ }^{\star} \star \star$}

\author{
J. Patience ${ }^{1,2}$, R. R. King ${ }^{1}$, R. J. De Rosa ${ }^{1}$, A. Vigan ${ }^{1}$, S. Witte ${ }^{3}$, E. Rice ${ }^{4, \star \star \star}$, Ch. Helling ${ }^{5}$, and P. Hauschildt ${ }^{3}$ \\ 1 Astrophysics Group, School of Physics, University of Exeter, Exeter, EX4 4QL, UK \\ e-mail: [patience;rob; derosa; arthur]@astro.ex.ac.uk \\ 2 ASU School of Earth and Space Exploration, PO Box 871404, Tempe, AZ 85287-1404, USA \\ 3 Hamburger Sternwarte, Gojenbergsweg 112, 21029 Hamburg, Germany \\ e-mail: [switte; yeti]@hs.uni-hamburg.de \\ 4 Department of Astrophysics, American Museum of Natural History, 79th Street and Central Park West, New York, NY 10024, USA \\ e-mail: erice@amnh.org \\ 5 SUPA, School of Physics \& Astronomy, University of St. Andrews, North Haugh, St. Andrews, KY16 9SS, UK \\ e-mail: ch80@st-andrews.ac.uk
}

Received 9 September 2011 / Accepted 5 January 2012

\section{ABSTRACT}

\begin{abstract}
With a uniform VLT SINFONI data set of nine targets, we have developed an empirical grid of $J, H, K$ spectra of the atmospheres of objects estimated to have very low substellar masses of $\sim 5-20 M_{\text {Jup }}$ and young ages ranging from $\sim 1-50$ Myr. Most of the targets are companions, objects which are especially valuable for comparison with atmosphere and evolutionary models, as they present rare cases in which the age is accurately known from the primary. Based on the youth of the sample, all objects are expected to have low surface gravity, and this study investigates the critical early phases of the evolution of substellar objects. The spectra are compared with grids of five different theoretical atmosphere models. This analysis represents the first systematic model comparison with infrared spectra of young brown dwarfs. The fits to the full $J H K$ spectra of each object result in a range of best fit effective temperatures of $\pm 150-300 \mathrm{~K}$ whether or not the full model grid or a subset restricted to lower $\log (g)$ values is used. This effective temperature range is significantly larger than the uncertainty typically assigned when using a single model grid. Fits to a single wavelength band can vary by up to $1000 \mathrm{~K}$ using the different model grids. Since the overall shape of these spectra is governed more by the temperature than surface gravity, unconstrained model fits did not find matches with low surface gravity or a trend in $\log (g)$ with age. This suggests that empirical comparison with spectra of unambiguously young objects targets (such as those presented here) may be the most reliable method to search for indications of low surface gravity and youth. Based on comparison with previous observations, the SINFONI spectra represent a second epoch for the targets $2 \mathrm{M} 0141$ and DH Tau B, and the combined data show no variations in the spectral morphology over time. The analysis of two other targets, AB Pic B and CT Cha B, suggests that these objects may have lower temperatures, and consequently lower masses, than previously estimated.
\end{abstract}

Key words. planetary systems - brown dwarfs - stars: atmospheres - binaries: close - techniques: high angular resolution

\section{Introduction}

Since the discovery of the first substellar companions GD 165B (Becklin \& Zuckerman 1988) and Gl 229B (Nakajima et al. 1995), there have been many surveys for additional low mass companions, but a very limited number have been discovered (e.g., McCarthy \& Zuckerman 2004; Metchev \& Hillenbrand 2006), particularly at the lowest masses (Zuckerman \& Song 2009). Substellar objects that are companions to nearby stars and brown dwarfs are especially valuable for comparison with atmosphere and evolutionary models, as they present rare cases

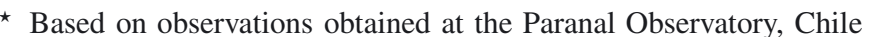
for ESO programs 279.C-5010(A), 080.C-0590(A), 077.C-0264(A), 078.C-0800(B), \& 078.C-0800(A).

$\star \star$ The spectra are only available at the CDS via anonymous ftp to cdsarc.u-strasbg.fr $(130.79 .128 .5)$ or via

http://cdsarc.u-strasbg.fr/viz-bin/qcat?]/A+A/540/A85

$\star \star \star$ Current address: Department of Engineering Science \& Physics, College of Staten Island, 2800 Victory Blvd, Staten Island, NY 10314, USA.
}

in which the brown dwarf age is inferred by the accurate age of the primary. Recent models (e.g., Fortney et al. 2008) have indicated the importance initial conditions may have in determining the flux from young objects, making confirmed young brown dwarfs important to gauge the effects of accretion history and atmospheric processes at low surface gravity.

The effects of low surface gravity on the infrared spectra of brown dwarfs have been investigated with isolated members of star-forming regions and young clusters. In the $J$-band, the alkali metal lines of $\mathrm{K} \mathrm{I}$ and $\mathrm{Na} \mathrm{I}$ and the $\mathrm{FeH}$ absorption were found to have lower equivalent widths in young brown dwarfs relative to field objects of the same spectral type in an initial sample of three young objects (McGovern et al. 2004) and a larger sample of 23 brown dwarfs in Upper Sco (Lodieu et al. 2008). Lower pressures in the atmospheres of the young objects cause a lower amount of recombination of $\mathrm{K}$ II and $\mathrm{Na}$ II to form $\mathrm{K} \mathrm{I}$ and $\mathrm{Na} \mathrm{I}$ which, along with pressure-broadening (Rice et al. 2010) and other processes, impacts the line strengths relative to the field objects, resulting in weaker lines. An index based on the $\mathrm{Na}$ I doublet was defined to assess surface gravity in the 
youngest objects (Allers et al. 2007). The triangular shape of the $H$-band was noted in the spectra of low luminosity $\sigma$ Orionis members (Lucas et al. 2001) and associated with a decrease in $\mathrm{H}_{2}$ collision induced absorption due to low gravity in a young field object (Kirkpatrick et al. 2006). Rice et al. (2011) demonstrate how the relative opacities from $\mathrm{CIA}-\mathrm{H}_{2}$ absorption and $\mathrm{H}_{2} \mathrm{O}$ at different gravities shape the spectra of young objects. The CO bandhead shape in the $K$-band has also been linked with lower surface gravity due to the sensitivity of the absorption to microturbulence, and has been used to differentiate dwarf and giant stars (Kleinmann \& Hall 1986).

Given the intrinsically low luminosities and temperatures of substellar objects, infrared observations provide higher signalto-noise spectra than optical measurements, making this wavelength range important for the characterization of the physical properties of brown dwarfs. For example, $J$-band spectra taken at two resolutions of a sample of M6-M9 objects including 15 brown dwarfs with ages $~ 1-10$ Myr were compared with a grid of model atmospheres to infer a number of properties - effective temperatures, surface gravities, radial velocities and projected rotational velocities (Rice et al. 2010).

This paper presents the combined flux-calibrated $J, H, K$ spectra for a set of young (2-50 Myr), low mass (5-25 $\left.M_{\text {Jup }}\right)$ substellar objects and compares the observations with a suite of synthetic spectra developed from five different theoretical model atmosphere simulations. The range of physical properties inferred from the different models is discussed, along with the discrepancies obtained from fits over portions of the $J, H, K$ wavelength range rather than the full combination. The spectral shape is the focus of this paper, and the investigation of spectral lines and longer wavelengths covering the $L$-band are the subject of a forthcoming paper (King et al., in prep.).

\section{Sample and summary of previous observations}

The sample consists of 9 objects estimated to have very low masses of $\sim 5-20 M_{\text {Jup }}$ and young ages ranging from $\sim 1-50 \mathrm{Myr}$, placing them at the boundary of the brown dwarf and planetary mass regimes. The sample covers three age bins, with three $\sim 1-$ 3 Myr old members of star-forming regions, three members of the $\sim 8$ Myr TW Hydra association, and three targets with ages of 30-50 Myr. Eight of the targets are members of binary systems discovered with adaptive optics imaging surveys, and one object is an isolated young brown dwarf. The youngest objects may still be surrounded by material from their formation environment and have the possibility of accretion and disks, while the older objects likely present bare photospheres. All are expected to have low surface gravity. The distinct position (dereddened, when necessary) on the color-magnitude diagram of this young, low surface gravity sample compared to field objects is shown in Fig. 1; the trend is as predicted in theoretical evolutionary models (e.g., Saumon \& Marley 2008). The targets show redder colors than field $\mathrm{L}$ and $\mathrm{T}$ dwarfs, analogous to the imaged planets orbiting HR 8799. For all members of the sample, there are archive infrared $J, H, K$ spectra from the VLT integral field spectrograph SINFONI, forming a uniform data set with which it is possible to construct an empirical grid of the atmospheres of substellar objects covering the critical early phases of their evolution.

\subsection{Star-forming region systems}

High angular resolution imaging surveys targeting members of the nearest star-forming regions have identified a number of

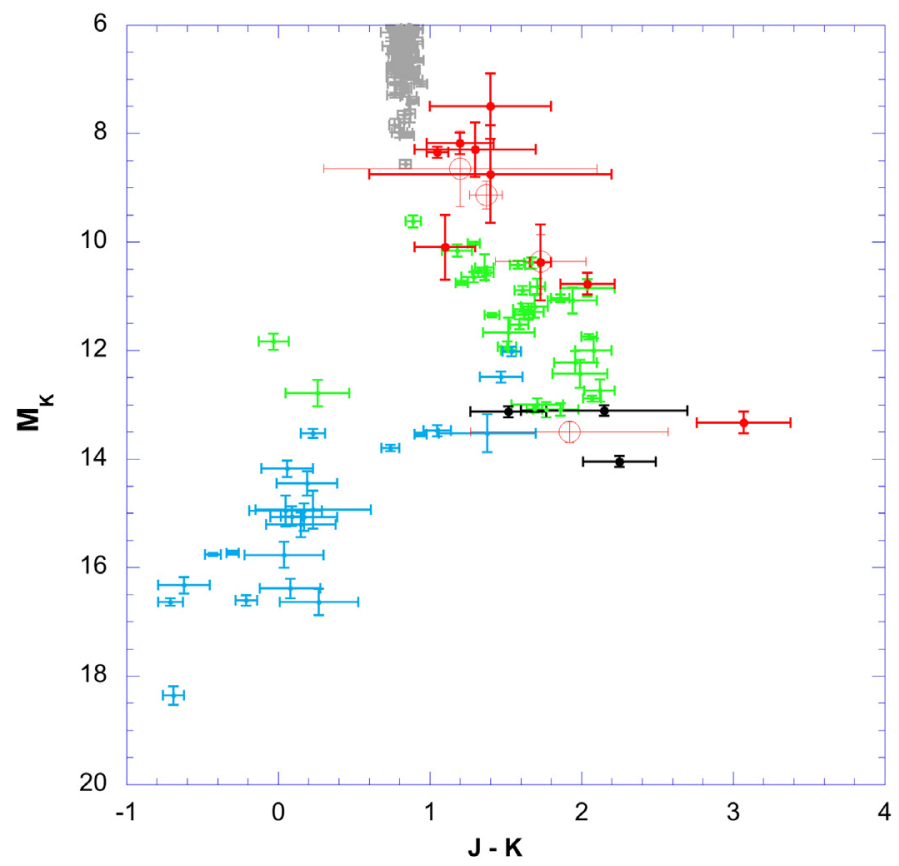

Fig. 1. Color-magnitude diagram of Field M (grey), L (green), T (blue) objects, the sample targets (filled red) and other young companions (unfilled red), and the HR 8799 planets with measured $J$ and $K$ magnitudes (black). The field data were taken from the DwarfArchive.org and restricted to objects with parallax uncertainty $<10 \%$ and color uncertainty $<0.5 \mathrm{mag}$. The values used for the targets are given in Tables 1 and 2 . For the young companions CHXR 73B (Luhman et al. 2006), 1RXS J1609B (Lafrenière et al. 2008), GSC 06214 B (Ireland et al. 2011) and HD 203030B (Metchev \& Hillenbrand 2006) that are plotted on the figure, but not included in this study, the data were taken from the discovery papers.

very young ( 1-2 Myr) wide orbit (>100 AU) low mass brown dwarf companions. The pre-Main Sequence sample considered for this paper are the three companions with archive SINFONI $J, H, K$ spectra: GQ Lup B (Neuhäuser et al. 2005), DH Tau B (Itoh et al. 2005), and CT Cha B (Schmidt et al. 2008). The closest companion is GQ Lup B, with a separation of 0 ' 7 , or $\sim 105 \mathrm{AU}$ at a distance of $150 \pm 20 \mathrm{pc}$ (Franco 2002), and has been the subject of considerable prior investigation into the inferred companion mass and evidence for ongoing accretion. Based on a comparison of theoretical models with photometry and $K$-band spectroscopy, the mass was initially estimated as 1-42 $M_{\text {Jup }}$ (Neuhäuser et al. 2005), while subsequent analysis of a larger wavelength range (e.g., Marois et al. 2007) revised the mass range to $10-20 M_{\text {Jup }}$. In Table 1 , the mass is listed as $24 \pm 12 M_{\text {Jup }}$ (Luhman et al. 2006), since this estimate applied the same approach to both GQ Lup B and another target DH Tau B. The SINFONI spectra reported in Seifahrt et al. (2007) suggested evidence for active accretion from the $\mathrm{Pa} \beta$ emission line, while independent $J, H, K$ spectra from OSIRIS (McElwain et al. 2007) and NIFS (Lavigne et al. 2009) do not confirm the line, which may have resulted from contamination from the primary.The SINFONI spectrum of GQ Lup is included in this paper for completeness.

The companion DH Tau B (Itoh et al. 2005) is in a wider orbit, at 2.'3 (330 AU) from the primary, making contamination from the host star unlikely. The estimated mass for the companion, $11_{-3}^{+10} M_{\text {Jup }}$, listed in Table 1 is based on the same model comparison applied to GQ Lup B (Luhman et al. 2006) and within the 5-40 $M_{\text {Jup }}$ range reported in Itoh et al. (2008). The 
Table 1. Sample.

\begin{tabular}{|c|c|c|c|c|c|c|c|c|c|c|}
\hline Source & Primary & Comp. & $\begin{array}{c}\text { Age } \\
(\mathrm{Myr}) \\
\end{array}$ & $\begin{array}{l}M_{\text {prim }} \\
\left(M_{\odot}\right) \\
\end{array}$ & $\begin{array}{c}M_{\text {sec }} \\
\left(M_{\text {Jup }}\right)\end{array}$ & $\begin{array}{c}D \\
(\mathrm{pc}) \\
\end{array}$ & $\begin{array}{l}\text { Sep. } \\
\text { (AU) }\end{array}$ & Membership & $\overline{A_{J}}$ & Refs. \\
\hline GQ Lup B & K7 & L1.5 & 1 & 0.7 & $24 \pm 12$ & $150 \pm 20$ & 100 & Lupus SFR & $0.1 \pm 0.2$ & $1-5$ \\
\hline DH Tau B & M0.5 & L2 & 1 & 0.33 & $11 \pm{ }_{3}^{10}$ & $140 \pm 15$ & 330 & Taurus SFR & $0.3 \pm 0.3$ & 6,2 \\
\hline CT Cha B & K7 & $\geq \mathrm{M} 8$ & 2 & 0.7 & $17 \pm 6$ & $165 \pm 30$ & 440 & Chameleon SFR & $0.4 \pm 0.2$ & $7-8$ \\
\hline 2M1207 A\&B & M8 & L5 & 8 & $25 \pm 5 M_{\text {Jup }}$ & $6-10$ & $52.4 \pm 1.1$ & 46 & TW Hydra assoc. & - & $9-12$ \\
\hline TWA 5 B & M1.5 & M8/8.5 & 8 & 0.40 & $\sim 20$ & $44 \pm 4$ & 98 & TW Hydra assoc. & - & $13-15$ \\
\hline AB Pic B & $\mathrm{K} 2$ & L0-L1 & 30 & 0.84 & $13-14$ & $47.3 \pm 1.8$ & 248 & Tuc-Hor assoc. & - & $16-17$ \\
\hline GSC08047 B & K3 & M9.5 & 30 & 0.80 & $25 \pm 10$ & $\sim 85$ & 279 & Tuc-Hor assoc. & - & $18-19$ \\
\hline 2M0141 & L0 & - & $1-50$ & $15 \pm 10 M_{\text {Jup }}$ & - & $\sim 35$ & - & Tuc-Hor/ $\beta$ Pic? & - & 20 \\
\hline
\end{tabular}

References. (1) Neuhäuser et al. (2005); (2) Luhman et al. (2006); (3) Marois et al. (2007); (4) Franco (2002); (5) Batalha et al. (2001); (6) Itoh et al. (2005); (7) Schmidt et al. (2008); (8) Luhman (2004); (9) Gizis (2002); (10) Chauvin et al. (2004); (11) Chauvin et al. (2005a); (12) Mohanty et al. (2007); (13) Lowrance et al. (1999); (14) Webb et al. (1999); (15) Konopacky et al. (2007); (16) Chauvin et al. (2005c); (17) Bonnefoy et al. (2010); (18) Chauvin et al. (2005b); (19) Neuhäuser \& Guenther (2004); (20) Kirkpatrick et al. (2006).

Table 2. Photometry.

\begin{tabular}{lcccc}
\hline \hline Source & & $J$ & $H$ & $K$ \\
\hline CT Cha & A & $9.72 \pm 0.02$ & $8.94 \pm 0.05$ & $8.66 \pm 0.02$ \\
& B & $16.6 \pm 0.3$ & $\ldots$ & $14.9 \pm 0.3$ \\
DH Tau & A & $9.77 \pm 0.02$ & $8.82 \pm 0.03$ & $8.18 \pm 0.03$ \\
& B & $15.71 \pm 0.05$ & $14.96 \pm 0.04$ & $14.19 \pm 0.02$ \\
GQ Lup & A & $8.69 \pm 0.04$ & $7.70 \pm 0.03$ & $7.10 \pm 0.02$ \\
& B & $14.90 \pm 0.11$ & $\ldots$ & $13.34 \pm 0.13$ \\
& & & & \\
2M1207 & A & $13.00 \pm 0.03$ & $12.39 \pm 0.03$ & $11.95 \pm 0.03$ \\
& B & $20.0 \pm 0.2$ & $18.09 \pm 0.21$ & $16.93 \pm 0.11$ \\
TWA 5 & Aa & $8.40 \pm 0.07$ & $7.69 \pm 0.04$ & $7.39 \pm 0.04$ \\
& Ab & $8.5 \pm 0.2$ & $7.79 \pm 0.05$ & $7.62 \pm 0.08$ \\
& B & $12.6 \pm 0.2$ & $12.14 \pm 0.06$ & $11.4 \pm 0.2$ \\
& & & & \\
AB Pic & A & $7.58 \pm 0.03$ & $7.09 \pm 0.03$ & $6.98 \pm 0.03$ \\
& B & $16.18 \pm 0.10$ & $14.69 \pm 0.10$ & $14.14 \pm 0.08$ \\
GSC08047 & A & $9.06 \pm 0.03$ & $8.53 \pm 0.06$ & $8.41 \pm 0.03$ \\
& B & $15.9 \pm 0.1$ & $15.45 \pm 0.02$ & $14.8 \pm 0.1$ \\
2M0141 & & $14.83 \pm 0.04$ & $13.88 \pm 0.03$ & $13.10 \pm 0.03$ \\
\hline
\end{tabular}

previous CISCO $J, H, K$ spectrum $(R \sim 440)$ showed absorption from $\mathrm{H}_{2} \mathrm{O}$ bands indicating a low effective temperature, and no evidence of emission lines from accretion was present (Itoh et al. 2005). The SINFONI data presented in this paper were taken at higher resolution $(R \sim 1500)$ than the CISCO spectrum and at a different epoch, enabling a search for variability.

The widest young companion CT Cha B is 2.'7 (440 AU) from the primary (Schmidt et al. 2008). Based on fitting the SINFONI $J, H, K$ spectra with Drift-PHOENIX models to determine the temperature and photometry to estimate the luminosity, the companion mass was estimated to be $17 \pm 6 M_{\text {Jup }}$ (Schmidt et al. 2008) from comparison with evolutionary models (Baraffe et al. 2003; Chabrier et al. 2000; Burrows et al. 1997). The spectrum shows $\mathrm{Pa} \beta$ emission, an indication of ongoing accretion, though another accretion diagnostic $\mathrm{Br} \gamma$ is not seen (Schmidt et al. 2008). In this paper, we reconsider the SINFONI spectra in comparison with a larger set of models and with a different treatment of extinction.

\subsection{TW Hydra association systems}

Two members of the TW Hydra association - TWA 5 and 2M1207 - include three substellar objects, with both late-M and mid-L spectral types. TWA 5B was one of the earliest brown dwarf companions identified (Lowrance et al. 1999; Webb et al. $1999)$ and is located $2 . \prime 0$ ( 100 AU) from a close pair of early M-stars with a $\sim 5$ yr orbit (Konopacky et al. 2007). Although the photometry of TWA 5B has been explored (Neuhäuser et al. 2000), the $H$ - and $K$-band spectra have been published separately (Neuhäuser et al. 2009). The second TW Hydra member, 2M1207, is composed of two substellar objects (Chauvin et al. 2004, 2005a) - the primary is an M8 brown dwarf (Gizis 2002) similar to TWA 5B and the secondary is a L-type planetary mass object (Chauvin et al. 2004; Mohanty et al. 2007). The complete $J, H, K$ spectrum of 2M1207B has been published (Patience et al. 2010), but not the corresponding spectrum of the primary. When compared with DUSTY models, the companion spectrum presents a spectral shape consistent with temperatures higher than expected from the luminosity (Mohanty et al. 2007; Patience et al. 2010). The discrepancy between spectral shape and flux level was initially explained by obscuration from a grey disk (Mohanty et al. 2007), and then updated to include models incorporating an improved treatment of clouds (Skemer et al. 2011) and both clouds and non-equilibrium chemistry (Barman et al. 2011) to simultaneously fit the spectrum shape and flux level. The 2M1207 B spectrum represents an example of how measurements of young, low mass object atmospheres can advance the understanding of the atmospheric physics.

\subsection{Tucana-Horolgium systems}

An additional two substellar companions - AB Pic B and GSC 080647 B - are members of the older 30 Myr TucanaHorologium association. AB Pic B is a wide orbit 5.5 ( $260 \mathrm{AU}$ ) common proper motion companion (Chauvin et al. 2005c) to a K2 primary (Perryman et al. 1997). Based on a comparison of the system photometry with evolutionary models (Burrows et al. 1997; Baraffe et al. 2002) at $30 \mathrm{Myr}$, the mass of the companion is estimated to be 13-14 $M_{\text {Jup }}$ (Chauvin et al. 2005c), placing AB Pic B at the demarcation of planetary masses and brown dwarf masses. The $J, H, K$ SINFONI spectra have been used to estimate a spectral type for the companion of L0-L1 and to compare each band of the spectrum 
Table 3. SINFONI observations.

\begin{tabular}{|c|c|c|c|c|c|c|c|c|}
\hline Source & Grating & Scale & NDIT & DIT & \#Exp. & Dates & ESO Project ID & PI \\
\hline CT Cha B & $J$ & 100 mas & 2 & $138.5 \mathrm{~s}$ & 6 & 18 May 07 & 279.C-5010(A) & Schmidt \\
\hline CT Cha B & $H+K$ & 100 mas & 2 & $138.5 \mathrm{~s}$ & 6 & 16 May 07 & 279.C-5010(A) & Schmidt \\
\hline DH Tau B & $J$ & $25 \mathrm{mas}$ & 1 & $300 \mathrm{~s}$ & 32 & 18 Dec. 07 & 080.C-0590(A) & Rojo \\
\hline DH Tau B & $H+K$ & 25 mas & 1 & $300 \mathrm{~s}$ & 8 & 22 Oct. 07 & 080.C-0590(A) & Rojo \\
\hline GQ Lup B & $J$ & $25 \mathrm{mas}$ & 1 & $300 \mathrm{~s}$ & 27 & 18 Sep. 06 & 077.C-0264(A) & Neuhäuser \\
\hline GQ Lup B & $H$ & 25 mas & 1 & $300 \mathrm{~s}$ & 11 & 24 Apr. 06 & 077.C-0264(A) & Neuhäuser \\
\hline GQ Lup B & K & 25 mas & 1 & $300 \mathrm{~s}$ & 8 & 17 Sep. 05 & 077.C-0264(A) & Neuhäuser \\
\hline TWA 5B & $J$ & 25 mas & 1 & $120 \mathrm{~s}$ & 6 & 12 Dec. 07 & 080.C-0590(A) & Rojo \\
\hline TWA 5B & $H+K$ & 25 mas & 2 & $60 \mathrm{~s}$ & 7 & 13 Dec. 07 & 080.C-0590(A) & Rojo \\
\hline $2 \mathrm{M} 1207 \mathrm{~A}+\mathrm{B}$ & $J$ & $100 \mathrm{mas}$ & 1 & $600 \mathrm{~s}$ & 22 & 25 Feb. 07 & 078.C-0800(B) & Thatte \\
\hline $2 \mathrm{M} 1207 \mathrm{~A}+\mathrm{B}$ & $H+K$ & 100 mas & 1 & $300 \mathrm{~s}$ & 24 & 28 Jan. 07, 07Feb. 07 & 078.C-0800(B) & Thatte \\
\hline AB Pic B & $J$ & 25 mas & 1 & $300 \mathrm{~s}$ & 40 & 5-7 Dec. 07 & 080.C-0590(A) & Rojo \\
\hline AB Pic B & $H+K$ & 25 mas & 1 & $300 \mathrm{~s}$ & 8 & 11 Nov. 07 & 080.C-0590(A) & Rojo \\
\hline GSC 08047 B & $J$ & 25 mas & 1 & $300 \mathrm{~s}$ & 24 & 6\&18 Jan. 08 & 080.C-0590(A) & Rojo \\
\hline GSC $08047 \mathrm{~B}$ & $H+K$ & 25 mas & 1 & $300 \mathrm{~s}$ & 27 & 5\&9 Jan. 08 & 080.C-0590(A) & Rojo \\
\hline 2M0141 & $J$ & $250 \mathrm{mas}$ & 1 & 300 & 4 & 19 Oct. 06 & 078.C-0800(A) & Thatte \\
\hline $2 \mathrm{M} 0141$ & $H+K$ & 250 mas & 1 & $60 \mathrm{~s}$ & 4 & 19 Oct. 06 & 078.C-0800(A) & Thatte \\
\hline
\end{tabular}

with theoretical atmosphere models (Bonnefoy et al. 2010). In this paper, a model comparison of the combined flux-calibrated $J-K$ spectrum is presented, and the combined spectrum shows differences with the models not apparent in the single band comparisons. Another Tucana-Horologium pair with very similar spectral types to AB Pic is GSC 08047, which is composed of a K3 primary with a confirmed substellar companion 3". 3 (279 AU) from the host star (Chauvin et al. 2005b). Previous spectra in the $H$ - and $K$-bands taken with VLT/ISAAC and VLT/NACO were compared with late-M objects to estimate a spectral type of M8 \pm 2 (Neuhäuser \& Guenther 2004) and M9.5 \pm 1 (Chauvin et al. 2005b), slightly earlier than the AP Pic companion. The new SINFONI spectra include the $J$-band and the IFU observations are not subject to wavelength-dependent effects of differences in the slit centering for target and standard that affected some of the previous spectra.

\subsection{Young isolated field object}

As part of the large scale search and characterization program for L-dwarfs with 2MASS, an isolated brown dwarf with indications of youth was identified with spectroscopy spanning $0.6-2.5 \mu \mathrm{m}$ (Kirkpatrick et al. 2006). In the infrared, there is a $J-K$ SpeX spectrum with similar resolution $(R \sim 1200)$ to the SINFONI data, and a NIRSPEC $J$-band spectrum with higher $(R \sim 2500)$ resolution, making $2 \mathrm{M} 0141$ an ideal case to test for variability such as has been reported for TWA 30 (Looper et al. 2010). For 2M0141, the spectral features of weak alkali lines such as K I in the $J$-band and the triangular shape of the $H$-band caused by a decrease in $\mathrm{H}_{2}$ collision induced absorption provided evidence of a low surface gravity object (Kirkpatrick et al. 2006). The effective temperature and surface gravity were derived from fits to atmospheric models generated with the PHOENIX code (Kirkpatrick et al. 2006), and $T_{\text {eff }}$ and $\log (g)$ were compared with evolutionary models (Baraffe et al. 2002) to infer an age range of 1-50 Myr and object mass of 6-25 Myr (Kirkpatrick et al. 2006). Although $2 \mathrm{M} 0141$ currently has a less certain age, future measurements of the parallax and space motion may determine membership in the Tucana-Horologium association or the $\beta$ Pic moving group. In this paper, the SINFONI spectra are compared with a larger set of atmosphere models and with the earlier SpeX spectrum to search for variability.

\section{SINFONI observations}

The observations were taken with the SINFONI (Spectrograph for INtegral Field Observations in the Near Infrared) instrument (Bonnet et al. 2004; Eisenhauer et al. 2003; Thatte et al. 1998), an AO-equipped integral field spectrograph mounted at the Cassegrain focus of the VLT (UT4). The target low mass companions are separated by less than a few arcseconds from the primaries, making the primaries ideal sources for the AO correction. The observing sequence for all targets included a set of exposures on the target with offset positions on the array. For some targets, separate blank sky exposures were also recorded. Standard calibration observations of an early-type or solar-type star followed each target sequence to correct for telluric features and the instrument response. Additional calibrations were obtained to measure the wavelength scale, the detector dark current, distortion, and quantum efficiency variations. The data for this project were drawn from the ESO archive, and a summary of the instrument configuration, observation details and project ID codes for each target is given in Table 3 . In some cases, only the subset of higher quality data were used; Table 3 lists only the observations used in this analysis.

There are four gratings available in SINFONI $-J, H, K$, and $H+K-$ which produce spectral resolutions of approximately 2000, 3000, 4000, and 1500, depending on the scale. For all targets except GQ Lup B, a combination of $J$ and $H+K$ observations were taken. For GQ Lup B, separate $J, H$, and $K$ spectra were recorded. Among the three available spaxel (spatial pixel) scales of 25 mas, 100 mas, and 250 mas, the 100 mas scale was employed for the CT Cha B and 2M1207A+B observations. The 100 mas scale provides a field-of-view of $3^{\prime \prime}\left(0 \times 33^{\prime \prime} 0\right.$, and both the brown dwarf primary and planetary mass secondary for $2 \mathrm{M} 1207$ were included within each observation. For the remaining target, the spaxel scale of 25 mas was used and the field-of-view at this scale is $0{ }^{\prime} 8 \times 00^{\prime} 8$ which excluded the primary host star from the observations. For one target, GQ Lup B, the diffraction spike from the primary intersected the position of the target companion in a subset of the observations. 


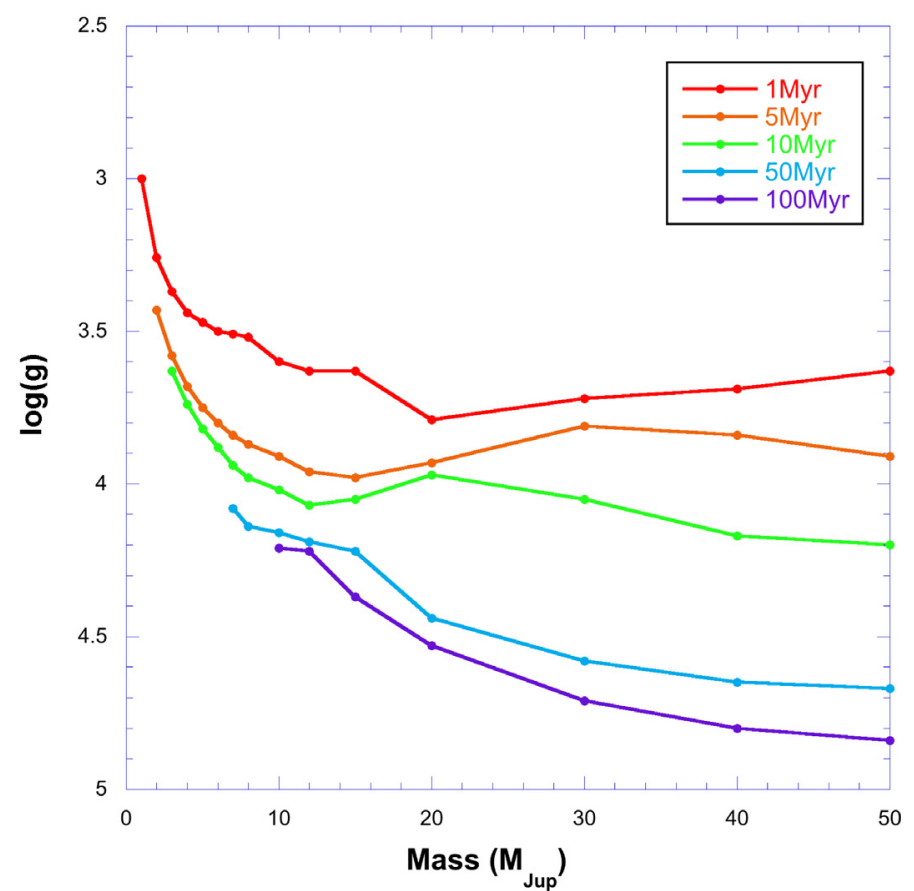

Fig. 2. The evolution in surface gravity over the 1-100 Myr age range for objects with masses of $\sim 1-50 M_{\text {Jup }}$. The values are taken from the DUSTY00 models (Chabrier et al. 2000) and show the systematic increase in $\log (g)$ over time as the objects contract. These tracks are used to determine the restricted range of surface gravity to allow in model fits to our young targets.

\section{Data analysis}

\subsection{SINFONI data reduction}

The initial steps of the data reduction for all targets were performed with the Gasgano (Gebbinck et al. 2007) implementation of the SINFONI data reduction pipeline (Modigliani et al. 2009; Dumas et al. 2007). The processing began with the raw data and calibration files in the ESO archive and is independent of previously reported results summarised in Sect. 2. This approach was taken to ensure that all data used the same software version and were reduced in a consistent way. Corrections for the dark current, linearity response, and bad pixels were measured and applied to all data files. The instrument optical distortion was measured with a set of fibers illuminating positions across the field and the non-linear wavelength dispersion was measured with observations of an arc lamp; both the spatial and spectral calibrations were applied to the data with Gasgano routines. The calibrated two-dimensional data for each observation was converted into a three-dimensional data cube consisting of an image at each wavelength slice. Across the entire wavelength range, a stack of 700-2000 wavelength slices images were recorded, however the faintest target $2 \mathrm{M} 1207 \mathrm{~B}$ required binning in the $J$-band to produce a spectrum with a higher signal-to-noise ratio.

After corrections for the detector and optics, the sky background emission was subtracted from the offset position covering either blank sky or including the target at a different position on the array in the ABBA pattern of observations. The individual sky-subtracted cubes were aligned based on centroiding the target in the top wavelength slice and then combined. To ensure the final spectrum traced a straight path through the data cube, each wavelength slice of the final data cube was shifted and aligned, and then the final spectrum was extracted. For the one target with both components on the array, 2M1207, it was

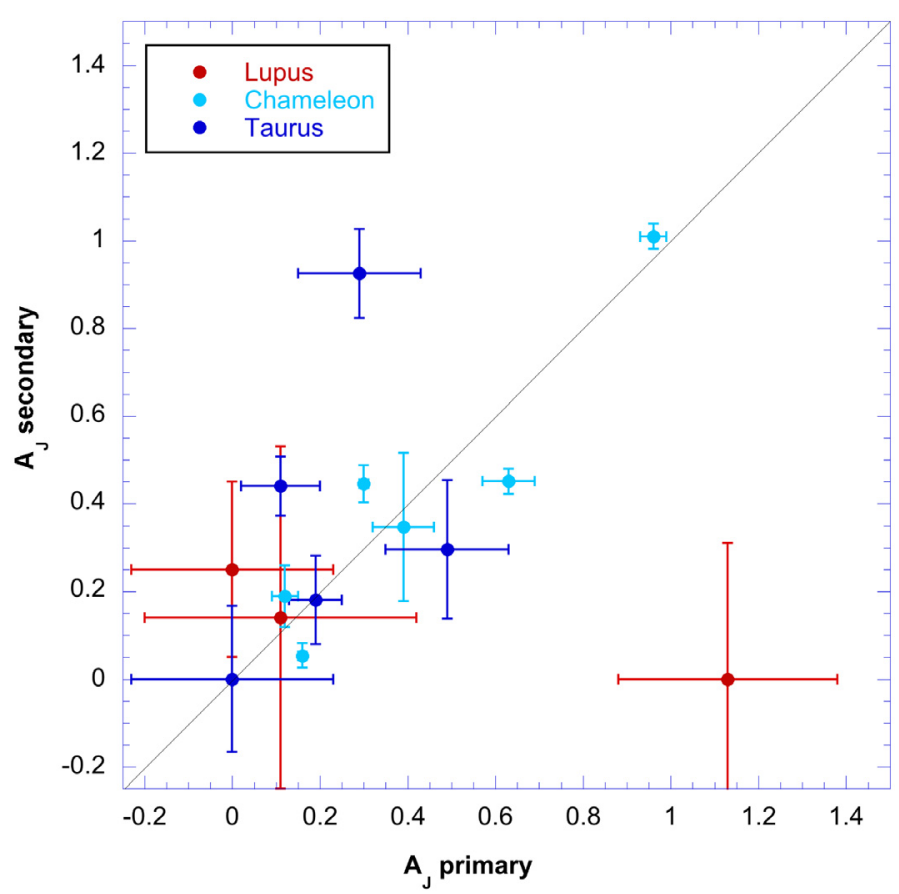

Fig. 3. The extinction $A_{J}$ of the secondary as a function of the $A_{J}$ of the primary for spatially resolved binary systems in Lupus (red), Chameleon (light blue), and Taurus (dark blue) based on observations of the individual components of each system (Brandner \& Zinnecker 1997; White \& Ghez 2001; Prato et al. 2003). These binaries have comparable separations to the target systems and are located in the same star-forming regions. The data show a trend of comparable extinction for each component.

necessary to subtract the halo of the primary before extracting the spectrum of the companion. Further details of the subtraction have been reported previously (Patience et al. 2010).

The standard stars observed after the targets were used to correct for telluric absorption and the wavelength response of the detector by dividing each target spectrum by its associated standard after interpolating the standard spectrum over intrinsic features. The shape of the standard star continuum was removed by multiplication of a black body with the same effective temperature as the standard. To flux calibrate the spectra, it was necessary to rely on photometric measurements presented in Table 2 which were not contemporaneous with the spectra. The observed spectra and the spectrum of Vega (Mountain et al. 1985; Hayes 1985) were convolved with the 2MASS response curves (Carpenter 2001) to calculate the scaling factor required to match the reported magnitude in a given filter. For the $H+K$ spectra, the $H$-band photometry was used for flux calibration, because the SINFONI data covers the full 2MASS $H$-band filter bandpass. For one target, GQ Lup B, there is no reported $H$-band magnitude and the $H$ and $K$ spectra were observed separately. In this case, the $H$-band magnitude was estimated by assuming the same $H-K$ color as DH Tau B, since GQ Lup B and DH Tau B both have a very similar $J-K$ color and similar young ages. Another target, CT Cha B, also had no $H$-band measurement, and, in this case, the $K$-band magnitude was used to flux calibrate the $H+K$ spectrum.

\subsection{Comparison with theoretical model grids}

The target spectra were compared with five sets of theoretical models, spanning a range of effective temperature and 

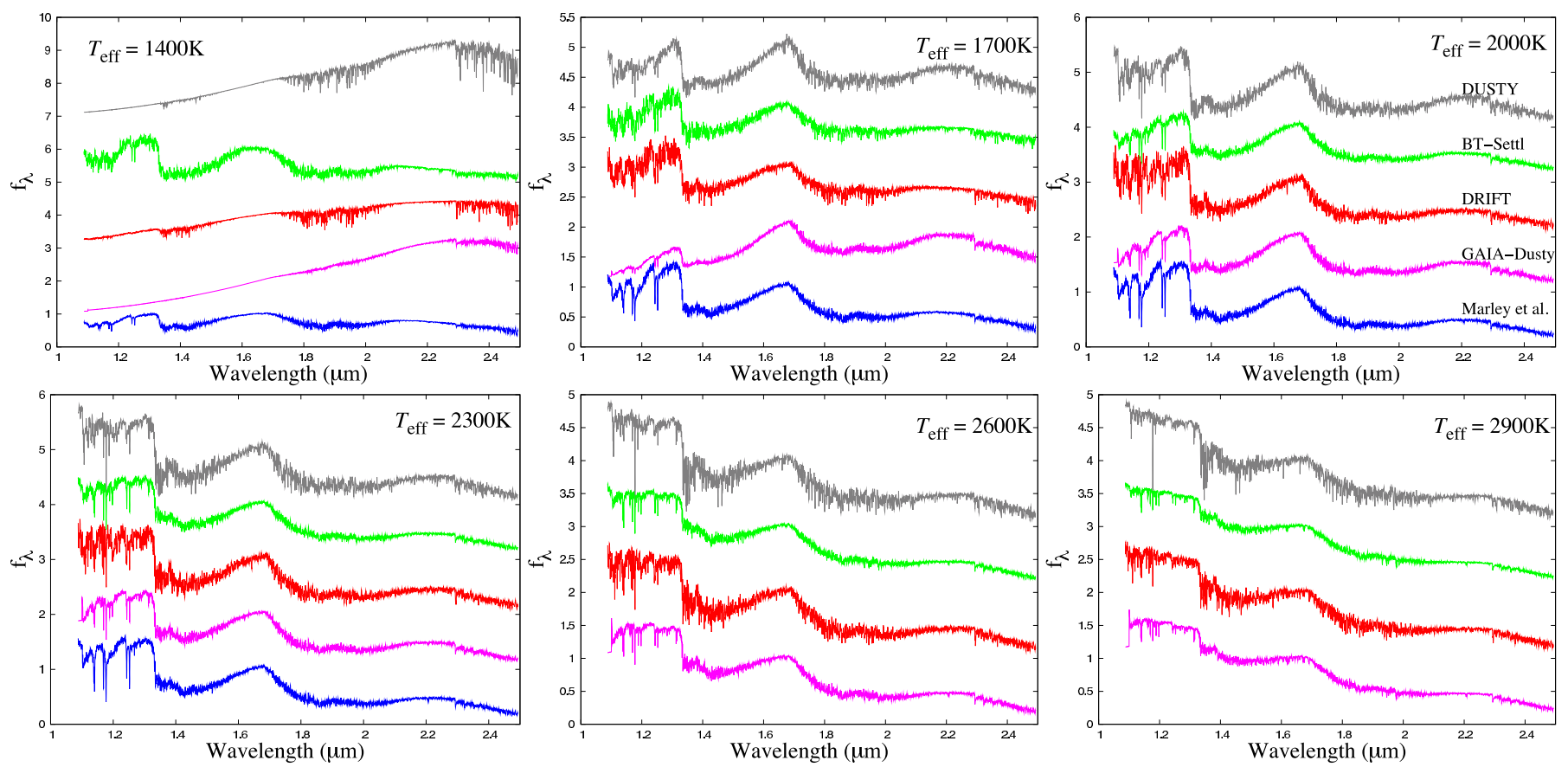

Fig. 4. For a fixed value of $\log (g)=4.5$, the solar metallicity model spectra at temperatures ranging from $1400 \mathrm{~K}$ (upper left) to $2900 \mathrm{~K}$ (lower right) in steps of $300 \mathrm{~K}$, corresponding to the target effective temperatures. From top to bottom in each panel, the models are: DUSTY (grey), BT-Settl (green), Drift-PHOENIX (red), Gaia-Dusty (purple), and Marley et al. (blue). The Marley et al. models do not include temperatures above $2400 \mathrm{~K}$, and the plots show models with $f_{\text {sed }}=1$, the dustiest set of models within this model grid.

surface gravity, as described in Sect. 5. The model spectra were smoothed to the same resolution as the data and interpolated to the same dispersion before performing a fitting procedure using the least-squares statistic defined in Mohanty et al. (2007) and allowing the model flux to be scaled to find the best fit. This flux scaling is related to the object radius, enabling an estimate of the radius in addition to the effective temperature and surface gravity. Since each wavelength step in the SINFONI spectra is nearly constant over the $J H K$ range, we do not employ the weighting technique of Cushing et al. (2008) which was developed for spectra covering a much larger range of wavelengths.

Two sets of fits were performed to the model grids - a fit including the full range of $\log (g)$ values and a fit restricted to $\log (g) \geq 3.0$ and with an upper limit based on evolutionary models (Chabrier et al. 2000) shown in Fig. 2. The targets have known young ages, so the predicted evolution in surface gravity was used to set $\log (g)$ limits: 4.0 for the companions to pre-Main Sequence stars, 4.5 for the TW Hydra members, and 5.0 for the 30-50 Myr targets.

A final factor affecting the slope of the observed spectrum is the amount of extinction to each target. This is particularly important for the three objects in star-forming regions. None of the secondaries have measured values of extinction, however, the primaries have reported $A_{V}$ values. Rather than treat extinction as a free parameter, we use the results of binary star component extinction comparisons to gauge the likelihood of very different $A_{J}$ values for the primary and secondary. The secondary $A_{J}$ is shown as a function of primary $A_{J}$ in Fig. 3 for binary stars in the same regions as the targets (Brandner \& Zinnecker 1997; White \& Ghez 2001; Prato et al. 2003), and a clear trend of similar extinction values is seen. Based on the binary stars, the targets in this sample are assumed to have the same extinction as the primary, which is reported in Table 1.

\section{Theoretical models}

The data were compared with five grids of theoretical atmosphere models. Four of the models represent different extensions of the PHOENIX (Hauschildt et al. 1999) code and incorporate a range of dust properties and physical effects. In chronological order of development, the PHOENIX-based models considered in this paper include: DUSTY (Allard et al. 2001), BT-Settl (Allard et al. 2003, 2010), Drift-PHOENIX (Helling et al. 2008a; Witte et al. 2009), and Gaia-Dusty (Rice et al. 2010), with the latter three representing ongoing modelling efforts. The earlier generation DUSTY model is included for comparison, although the BTSettl and Gaia-Dusty models have superseded the DUSTY models with improved treatments of abundances and line lists. The independent model grid developed by Marley et al. (Ackerman \& Marley 2001; Marley et al. 2003; Saumon \& Marley 2008; Freedman et al. 2008) is also compared with the target spectra.

A summary of the physics incorporated into the five models is given in Table 4; details of the models are given in the references listed above and the comparison of models with two benchmark test cases is reported in (Helling et al. 2008b). The treatment of some physical processes such as convection is identical for all models, while the approach to other factors such as condensate settling and chemistry varies between the models. Since dust plays a large role in determining the temperaturepressure structure of the atmospheres in the temperature regime appropriate for the targets, the number of dust species and dust size distributions involved in the calculations are listed, along with notes on the mixing and settling included in the models.

An example of the synthetic spectra produced by each model over the same range of $T_{\text {eff }}$ for a fixed value of $\log (g)=4.5$ is given in Fig. 4. This value of $\log (g)$ was selected because it is within the range predicted by evolutionary models for the ages of the targets. For the Marley et al. spectra, the value of $f_{\text {sed }}$ is 1 which represents the dustiest atmospheres. At the coolest 
Table 4. Cloud models ${ }^{a}$.

\begin{tabular}{lccccc}
\hline \hline Physics & DUSTY & BT-Settl & Drift-PHOENIX & Gaia-Dusty & Marley et al. \\
\hline Opacity source & 30 dust species & 43 dust species & 7 dust species & updated DUSTY & 5 dust species \\
Gas-dust phase & $S=1$ & $S=1.001$ & $S=f(z, s)$ & $S=1$ & $S=1$ \\
Mixing & none & timescale from RHD & damping of turnover timescale & none & diffusive \\
Settling/Rainout & none & timescale comparison & equation of motion & none & parameterised in $f_{\text {sed }}$ \\
Dust sizes & $f(a) \propto a^{-3.5},(\mathrm{ISM})$ & timescale comparison & $f(a, z)^{d}$ & $f(a) \propto a^{-3.5},(\mathrm{ISM})$ & log-normal $f(z, s)^{e}$ \\
& $0.00625-0.24 \mu \mathrm{m}$ & $0.00625-0.24 \mu \mathrm{m}$ & $10^{-6}-100 \mu \mathrm{m}$ & $0.00625-0.24 \mu \mathrm{m}$ & $10^{-5}-1 \mu \mathrm{m}$ \\
Chemistry & equilibrium & equilibrium & equilibrium & equilibrium ${ }^{f}$ & equilibrium except \\
& & & & $\mathrm{CO}^{f} \mathrm{CH}_{4}, \mathrm{~N}_{2} / \mathrm{NH}_{3}$ \\
\hline
\end{tabular}

Notes. ${ }^{(a)}$ PHOENIX (Hauschildt et al. 1999) DUSTY (Allard et al. 2001), BT-Settl (Allard et al. 2003, 2010), Drift-PHOENIX (Helling et al. 2008a; Witte et al. 2009), Gaia-Dusty (Rice et al. 2010), Marley et al. (Ackerman \& Marley 2001; Marley et al. 2003; Saumon \& Marley 2008; Freedman et al. 2008). ${ }^{(b)} S$ quantifies the supersaturation ratio. $S=1$ for thermal equilibrium between the gas and dust. ${ }^{(c)}$ Parameterised in $k_{z z}$. (d) $f(a, z)$ is the grain size distribution at height $z$ and provides the number of grains of size $a .{ }^{(e)} f(z, s)$ is the grain size distribution for a grain species $s$ at height $z .{ }^{(f)}$ Updated chemistry treatment compared to DUSTY.

Table 5. Model grid parameters.

\begin{tabular}{lrcccc}
\hline \hline Model & \multicolumn{4}{c}{$T_{\text {eff }}$} & \multicolumn{2}{c}{$\log (g)$} & $\Delta \lambda$ \\
& range & step & range & step & \\
\hline DUSTY & $500-4000$ & 100 & $3.5-6.0$ & 0.5 & $2 \AA$ \\
BT-Settl $^{a}$ & $400-3000$ & 100 & $3.5-5.5$ & 0.5 & $0.2 \AA$ \\
Drift-PHOENIX & $1000-3000$ & 100 & $3.0-5.5$ & 0.5 & $2 \AA$ \\
Gaia-Dusty & $1400-3500$ & 50 & $3.0-6.5$ & 0.1 & $0.5 \AA$ \\
Marley et al. $^{b}$ & $900-2400$ & 100 & $4.0-5.0$ & 0.25 & $R \sim 50000$ \\
\hline
\end{tabular}

Notes. ${ }^{a}$ For $T_{\text {eff }}=2000-3000 \mathrm{~K}$, the $\log (g)$ range is $0.0-5.5$. ${ }^{b}$ The wavelength step $\Delta \lambda$ is a function of $\lambda$.

temperature shown, $1400 \mathrm{~K}$, there is a significant difference in the synthetic shape of the $H$-band spectrum and the overall slope for the different models. These effects are reduced by $1700 \mathrm{~K}$, but still impact the peak of the $J$-band. By a temperature of $2000 \mathrm{~K}$, the slope across the $J$-band ranges from flat to increasing. At hotter temperatures, the line strengths differentiate the models more than the overall shape, and the atomic and metal hydride lines will be investigated for this sample in a future paper (King et al., in prep.).

These model differences are primarily caused by the different treatments of condensate settling and the differences in dust opacity. Typically, a higher overall dust opacity results in a relatively lowered $J$-band flux and weakens the water bands while smoothing their edges. The $H$ band shape is dominated by the two adjacent water bands and the collision induced absorption (CIA) which both grow stronger with decreasing dust opacities as flux emerges from the deeper, hotter atmosphere resulting in a bluer spectrum.

Another difference between the models is apparent upon close comparison of the strong absorption lines and bands between models calculated at different resolutions. In regions of strong, varied absorption, models calculated at lower resolution (2 A) look "noisier", i.e. a larger spread in the flux values over small wavelength ranges (see Fig. 4). For example, the DUSTY models show the largest "noise" of the spectrum over the full temperature range, while the Marley et al., BT-Settl, and GaiaDusty models are typically much narrower in the range of flux at a given wavelength. These differences are a numerical artifact induced when the convolution with a Gaussian instrument dispersion profile is undersampled, resulting in lower fidelity model spectra for DUSTY and Drift-PHOENIX. The numerical origin of the difference was confirmed by calculating one grid
(Gaia-Dusty) with two initial resolutions and comparing the results. Due to the fact that the model fits in this paper are based only on the spectral shape, the additional width in the DUSTY and Drift-PHOENIX models does not impact this analysis, however the future comparison of spectral lines and indices requires models with sufficient resolution to fit line strengths.

Table 5 shows the range of $T_{\text {eff }}$ and $\log (g)$ covered by the solar metallicity models. The Marley et al. grids also incorporate a parameter $f_{\text {sed }}$ describing the sedimentation efficiency, and the values considered for $f_{\text {sed }}$ in this grid are 1 and 2, with the higher value associated with more efficient sedimentation from more rapid particle growth and larger average particle sizes. In addition to the solar metallicity models summarized in Table 5, the Drift-PHOENIX grid also covered non-solar metallicities with values of $-0.6,-0.3,0.0$, and +0.3 . The data are first compared with the full grid summarized in Table 5 and then with a restricted subset with limits on the $\log (g)$ values appropriate to the ages of the targets, based on evolutionary models (Chabrier et al. 2000). Finally, the impact of metallicity is explored with a comparison of the data with the expanded grid with different metallicities for the Drift-PHOENIX model.

\section{Results and discussion}

\subsection{SINFONI Spectra}

The $J, H, K$ flux-calibrated SINFONI spectra for the nine young substellar objects in the sample are plotted in Fig. 5, grouped in the three age bands and ordered in decreasing temperature determined from the model comparisons described in Sect. 6.2. Approximately half of the SINFONI data are previously unpublished: all wavelength bands of DH Tau B, 2M1207B, and 2M0141 are newly reported along with the $J$ - and $H$-bands of TWA 5B and GSC 080647 B. For the youngest targets, the spectra have been dereddened according to the reddening law of Cardelli et al. (1989) using the extinction measured for the primary, as described in Sect. 4.

The overall spectral shape is dominated by the $\mathrm{H}_{2} \mathrm{O}$ absorption at both sides of each wavelength band and the $\mathrm{CO}$ absorption band at the red edge of the $K$-band. All the object spectra exhibit a distinct triangular shape in the $H$-band portion of the spectrum, a signature of young substellar objects. As noted in other spectra of young objects, the peak of the $K$-band also occurs at a longer wavelength near $2.3 \mu \mathrm{m}$ than is typical for older field L-dwarfs (Mohanty et al. 2007). Two of the coolest objects, 2M1207 B and AB Pic B, show an unusually depressed $J$-band 
Table 6. Best fitting atmopshere model parameters, full grid comparison.

\begin{tabular}{|c|c|c|c|c|c|c|c|c|c|c|c|}
\hline \multirow[t]{2}{*}{ Wavelength range } & \multicolumn{2}{|c|}{ DUSTY } & \multicolumn{2}{|c|}{ BT-Settl } & \multicolumn{2}{|c|}{ Drift-PHOENIX } & \multicolumn{2}{|c|}{ Gaia-Dusty } & \multicolumn{3}{|c|}{ Marley et al. } \\
\hline & $T_{\text {eff }}(\mathrm{K})$ & $\log g$ & $T_{\text {eff }}(\mathrm{K})$ & $\log g$ & $T_{\text {eff }}(\mathrm{K})$ & $\log g$ & $T_{\text {eff }}(\mathrm{K})$ & $\log g$ & $T_{\text {eff }}(\mathrm{K})$ & $\log g$ & $f_{\text {sed }}$ \\
\hline \multicolumn{12}{|c|}{ 1-3 Myr objects } \\
\hline \multicolumn{12}{|c|}{ DH Tau B ${ }^{a}$} \\
\hline$J H K(1.10-2.46 \mu \mathrm{m})$ & 2600 & 4.0 & 2400 & 2.5 & 2000 & 4.0 & 2350 & 3.7 & $\geq 2400^{b}$ & 4.0 & 2 \\
\hline$J(1.10-1.34 \mu \mathrm{m})$ & 2400 & 4.0 & 2200 & 3.5 & 1600 & 3.5 & 2350 & 4.1 & $\geq 2400^{b}$ & 4.0 & 2 \\
\hline$H K(1.46-2.46 \mu \mathrm{m})$ & 2600 & 3.5 & 2500 & 3.5 & 1900 & 5.5 & 2550 & 3.8 & $\geq 2400^{b}$ & 4.0 & 2 \\
\hline \multicolumn{12}{|c|}{ GQ Lup B ${ }^{a}$} \\
\hline$J H K(1.10-2.46 \mu \mathrm{m})$ & 1900 & 6.0 & 1800 & 5.5 & 1700 & 3.5 & 2050 & 5.5 & 1600 & 4.5 & 1 \\
\hline$J(1.10-1.34 \mu \mathrm{m})$ & 2500 & 4.0 & 2400 & 3.5 & 2600 & 4.0 & 2500 & 4.8 & $\geq 2400^{b}$ & 4.0 & 2 \\
\hline$H K(1.46-2.46 \mu \mathrm{m})$ & 1800 & 6.0 & 1700 & 4.5 & 1700 & 5.0 & 1800 & 5.5 & 1500 & 4.5 & 1 \\
\hline \multicolumn{12}{|c|}{ CT Cha B ${ }^{a}$} \\
\hline$J H K(1.10-2.46 \mu \mathrm{m})$ & 1800 & 4.5 & 1600 & 4.0 & 1700 & 3.5 & 1950 & 4.1 & 1500 & 4.5 & 2 \\
\hline$J(1.10-1.34 \mu \mathrm{m})$ & 1900 & 3.5 & 1400 & 5.0 & 1700 & 3.0 & 2000 & 3.9 & 1100 & 5.0 & 2 \\
\hline$H K(1.46-2.46 \mu \mathrm{m})$ & 1900 & 6.0 & 2200 & 1.0 & 1800 & 5.0 & 1950 & 5.5 & 1700 & 4.5 & 1 \\
\hline \multicolumn{12}{|c|}{8 Myr objects } \\
\hline \multicolumn{12}{|c|}{ 2MASS 1207 A } \\
\hline$J H K(1.10-2.46 \mu \mathrm{m})$ & 3100 & 5.5 & 2800 & 5.0 & $\geq 3000^{b}$ & 5.0 & 2900 & 5.3 & $\geq 2400^{b}$ & 5.0 & 1 \\
\hline$J(1.10-1.34 \mu \mathrm{m})$ & 2900 & 5.0 & 2700 & 4.5 & $\geq 3000^{b}$ & 5.0 & 2850 & 5.1 & $\geq 2400^{b}$ & 4.0 & 2 \\
\hline$H K(1.46-2.46 \mu \mathrm{m})$ & 3100 & 5.5 & 2800 & 5.5 & $\geq 3000^{b}$ & 5.5 & 2950 & 5.5 & $\geq 2400^{b}$ & 5.0 & 1 \\
\hline \multicolumn{12}{|c|}{ TWA 5 B } \\
\hline$J H K(1.10-2.46 \mu \mathrm{m})$ & 2500 & 3.5 & 2400 & 2.5 & 2200 & 3.0 & 2450 & 3.3 & $\geq 2400^{b}$ & 4.0 & 2 \\
\hline$J(1.10-1.34 \mu \mathrm{m})$ & 2700 & 4.5 & 2600 & 4.0 & 2800 & 4.5 & 2700 & 5.0 & $\geq 2400^{b}$ & 4.0 & 2 \\
\hline$H K(1.46-2.46 \mu \mathrm{m})$ & 1800 & 6.0 & 2200 & 1.0 & 1800 & 5.5 & 1900 & 5.5 & 1600 & 4.5 & 1 \\
\hline \multicolumn{12}{|c|}{ 2MASS 1207 B } \\
\hline$J H K(1.10-2.46 \mu \mathrm{m})$ & 1600 & 3.5 & 1500 & 3.5 & 1500 & 5.0 & 1650 & 4.3 & 1100 & 5.0 & 1 \\
\hline$J(1.10-1.34 \mu \mathrm{m})$ & 1700 & 4.5 & 2000 & 0.5 & 1700 & 4.0 & 1950 & 4.0 & 1500 & 4.0 & 1 \\
\hline$H K(1.46-2.46 \mu \mathrm{m})$ & 1600 & 4.5 & 1500 & 3.5 & 1500 & 3.0 & 1650 & 4.7 & 1300 & 4.0 & 1 \\
\hline \multicolumn{12}{|c|}{ 30-50 Myr objects } \\
\hline \multicolumn{12}{|c|}{ GSC 08047 B } \\
\hline$J H K(1.10-2.46 \mu \mathrm{m})$ & 2200 & 3.5 & 2200 & 1.0 & 1900 & 4.5 & 2250 & 3.1 & 2300 & 4.0 & 2 \\
\hline$J(1.10-1.34 \mu \mathrm{m})$ & 2600 & 5.0 & 2200 & 3.5 & 1600 & 3.5 & 2300 & 4.2 & $\geq 2400^{b}$ & 4.0 & 2 \\
\hline$H K(1.46-2.46 \mu \mathrm{m})$ & 1800 & 6.0 & 1700 & 4.5 & 1700 & 4.0 & 1850 & 5.5 & 1600 & 4.5 & 1 \\
\hline \multicolumn{12}{|c|}{ 2MASS 0141} \\
\hline$J H K(1.10-2.46 \mu \mathrm{m})$ & 1800 & 5.0 & 2000 & 3.0 & 1700 & 4.0 & 2000 & 5.5 & 1600 & 4.5 & 1 \\
\hline$J(1.10-1.34 \mu \mathrm{m})$ & 2300 & 4.0 & 2200 & 3.5 & 1600 & 3.5 & 2300 & 4.9 & $\geq 2400^{b}$ & 4.0 & 2 \\
\hline$H K(1.46-2.46 \mu \mathrm{m})$ & 1800 & 6.0 & 2100 & 1.0 & 1800 & 5.0 & 1900 & 5.5 & 1600 & 4.5 & 1 \\
\hline \multicolumn{12}{|c|}{ AB Pic B } \\
\hline$J H K(1.10-2.46 \mu \mathrm{m})$ & 1800 & 6.0 & 1600 & 3.5 & 1600 & 4.5 & 1850 & 5.5 & 1400 & 4.5 & 1 \\
\hline$J(1.10-1.34 \mu \mathrm{m})$ & 2400 & 4.5 & 2300 & 4.0 & 1600 & 3.5 & 2400 & 4.9 & $\geq 2400^{b}$ & 4.0 & 2 \\
\hline$H K(1.46-2.46 \mu \mathrm{m})$ & 1900 & 6.0 & 2300 & 1.5 & 1800 & 5.0 & 1950 & 5.5 & 1700 & 5.0 & 1 \\
\hline
\end{tabular}

Notes. ${ }^{(a)}$ These star formation region members have been dereddened as described in Sect. $4 .{ }^{(b)}$ Note that this $T_{\text {eff }}$ falls at the edge of the grid used.

portion of the spectrum relative to the spectra of the other substellar objects of the same age.

\subsection{Comparison with atmosphere and evolutionary models}

\subsubsection{Full model grid}

Each object spectrum was compared with a grid of the five solarmetallicity models summarized in Sect. 5 - DUSTY, BT-Settl, Drift-PHOENIX, Gaia-Dusty, and Marley et al. The range in the parameters of $T_{\text {eff }}$ and $\log (g)$ are summarized in Table 5. The model fits were performed on each full $J, H, K$ spectrum and the $J$ and $H+K$ subsets to investigate the variation of inferred physical properties on the wavelength coverage of the data. The results of the model fits for $T_{\text {eff }}$ and $\log (g)$ are given in Table 6 , divided by the age groups and ordered by average $T_{\text {eff }}$ from the $J, H, K$ fit.

Based on the $J, H, K$ spectrum fits, the effective temperatures range from $1100 \mathrm{~K}$ to $3100 \mathrm{~K}$ and $\log (g)$ of 1.0 to 6.0 , considering the extremes of any given model fit. Some of the values for $\log (g)$ are outside the bounds expected from evolutionary models, and the fits also do not show a clear trend of increasing $\log (g)$ with increasing age. The lack of a clear correlation between $\log (g)$ and age may simply be a consequence of the coarseness of the grids and the dominant impact of temperature over surface gravity in shaping the overall spectral energy distribution, as opposed to specific gravity-sensitive features. The range of masses included in the sample also complicates the comparison of $\log (g)$ and age.

\subsubsection{Restricted $\log (g)$ model grid}

Since the targets all have well-constrained young ages, the spectra were also compared with a restricted model grid limited to $\log (g)$ values appropriate for each age range, as detailed in Sect. 4 and based on the evolutionary models shown in Fig. 2 (Chabrier et al. 2000). Further support for using a restricted $\log (g)$ grid comes from measurements of the $\mathrm{Na}$ index defined 
Table 7. Best fitting atmopshere model parameters, restricted grid comparison.

\begin{tabular}{|c|c|c|c|c|c|c|c|c|c|c|c|}
\hline \multirow{2}{*}{ Wavelength range } & \multicolumn{2}{|c|}{ DUSTY } & \multicolumn{2}{|c|}{ BT-Settl } & \multicolumn{2}{|c|}{ Drift-PHOENIX } & \multicolumn{2}{|c|}{ Gaia-Dusty } & \multicolumn{3}{|c|}{ Marley et al. } \\
\hline & $T_{\text {eff }}(\mathrm{K})$ & $\log g$ & $T_{\text {eff }}(\mathrm{K})$ & $\log g$ & $T_{\text {eff }}(\mathrm{K})$ & $\log g$ & $T_{\text {eff }}(\mathrm{K})$ & $\log g$ & $T_{\text {eff }}(\mathrm{K})$ & $\log g$ & $f_{\text {sed }}$ \\
\hline \multicolumn{12}{|c|}{$1-3$ Myr objects } \\
\hline \multicolumn{12}{|c|}{ DH Tau B ${ }^{a}$} \\
\hline$J H K(1.10-2.46 \mu \mathrm{m})$ & 2600 & 4.0 & 2400 & 3.0 & 2000 & 4.0 & 2350 & 3.7 & $\geq 2400^{b}$ & 4.0 & 2 \\
\hline$J(1.10-1.34 \mu \mathrm{m})$ & 2400 & 4.0 & 2200 & 3.5 & 1600 & 3.5 & 2350 & 4.0 & $\geq 2400^{b}$ & 4.0 & 2 \\
\hline$H K(1.46-2.46 \mu \mathrm{m})$ & 2600 & 3.5 & 2500 & 3.5 & 2700 & 4.0 & 2550 & 3.8 & $\geq 2400^{b}$ & 4.0 & 2 \\
\hline \multicolumn{12}{|c|}{ GQ Lup B ${ }^{a}$} \\
\hline$J H K(1.10-2.46 \mu \mathrm{m})$ & 1900 & 3.5 & 1900 & 3.5 & 1700 & 3.5 & 2050 & 3.5 & 1500 & 4.0 & 1 \\
\hline$J(1.10-1.34 \mu \mathrm{m})$ & 2500 & 4.0 & 2400 & 3.5 & 2600 & 4.0 & 2500 & 4.0 & $\geq 2400^{b}$ & 4.0 & 2 \\
\hline$H K(1.46-2.46 \mu \mathrm{m})$ & 1700 & 4.0 & 1700 & 3.5 & 1700 & 4.0 & 1750 & 4.0 & 1500 & 4.0 & 1 \\
\hline \multicolumn{12}{|c|}{ CT Cha B $^{a}$} \\
\hline$J H K(1.10-2.46 \mu \mathrm{m})$ & 1900 & 4.0 & 1600 & 4.0 & 1700 & 3.5 & 1950 & 4.0 & 1500 & 4.0 & 1 \\
\hline$J(1.10-1.34 \mu \mathrm{m})$ & 1900 & 3.5 & 1400 & 4.0 & 1700 & 3.0 & 2000 & 3.9 & 1000 & 4.0 & 1 \\
\hline$H K(1.46-2.46 \mu \mathrm{m})$ & 2500 & 3.5 & 2300 & 3.0 & 1800 & 4.0 & 2350 & 3.1 & 2400 & 4.0 & 2 \\
\hline \multicolumn{12}{|c|}{8 Myr objects } \\
\hline \multicolumn{12}{|c|}{ 2MASS $1207 \mathrm{~A}$} \\
\hline$J H K(1.10-2.46 \mu \mathrm{m})$ & 3200 & 4.0 & 2800 & 4.5 & $\geq 3000^{b}$ & 4.5 & 2900 & 4.5 & $\geq 2400^{b}$ & 4.5 & 1 \\
\hline$J(1.10-1.34 \mu \mathrm{m})$ & 2800 & 4.5 & 2700 & 4.5 & 2900 & 4.5 & 2800 & 4.5 & $\geq 2400^{b}$ & 4.0 & 2 \\
\hline$H K(1.46-2.46 \mu \mathrm{m})$ & 3100 & 4.5 & 3000 & 3.0 & $\geq 3000^{b}$ & 4.5 & 3050 & 4.5 & $\geq 2400^{b}$ & 4.5 & 1 \\
\hline \multicolumn{12}{|c|}{ TWA $5 \mathrm{~B}$} \\
\hline$J H K(1.10-2.46 \mu \mathrm{m})$ & 2500 & 3.5 & 2300 & 3.0 & 2200 & 3.0 & 2450 & 3.3 & $\geq 2400^{b}$ & 4.0 & 2 \\
\hline$J(1.10-1.34 \mu \mathrm{m})$ & 2700 & 4.5 & 2600 & 4.0 & 2800 & 4.5 & 2700 & 4.5 & $\geq 2400^{b}$ & 4.0 & 2 \\
\hline$H K(1.46-2.46 \mu \mathrm{m})$ & 2500 & 3.5 & 2000 & 3.0 & 1700 & 4.5 & 2250 & 3.1 & 1600 & 4.5 & 1 \\
\hline \multicolumn{12}{|c|}{ 2MASS 1207 B } \\
\hline$J H K(1.10-2.46 \mu \mathrm{m})$ & 1600 & 3.5 & 1500 & 3.5 & 1500 & 4.0 & 1650 & 4.3 & 1300 & 4.0 & 1 \\
\hline$J(1.10-1.34 \mu \mathrm{m})$ & 1700 & 4.5 & 2000 & 3.0 & 1700 & 4.0 & 1950 & 4.0 & 1500 & 4.0 & 1 \\
\hline$H K(1.46-2.46 \mu \mathrm{m})$ & 1600 & 4.5 & 1500 & 3.5 & 1500 & 3.0 & 1650 & 4.5 & 1300 & 4.0 & 1 \\
\hline \multicolumn{12}{|c|}{$30-50$ Myr objects } \\
\hline \multicolumn{12}{|c|}{ GSC $08047 \mathrm{~B}$} \\
\hline$J H K(1.10-2.46 \mu \mathrm{m})$ & 2200 & 3.5 & 2200 & 3.0 & 1900 & 4.5 & 2250 & 3.1 & 2300 & 4.0 & 2 \\
\hline$J(1.10-1.34 \mu \mathrm{m})$ & 2600 & 5.0 & 2200 & 3.5 & 1600 & 3.5 & 2300 & 4.2 & $\geq 2400^{b}$ & 4.0 & 2 \\
\hline$H K(1.46-2.46 \mu \mathrm{m})$ & 1700 & 5.0 & 1700 & 4.5 & 1700 & 4.0 & 1750 & 5.0 & 1600 & 4.5 & 1 \\
\hline \multicolumn{12}{|c|}{ 2MASS 0141} \\
\hline$J H K(1.10-2.46 \mu \mathrm{m})$ & 1800 & 5.0 & 2000 & 3.0 & 1700 & 4.0 & 2000 & 5.0 & 1600 & 4.5 & 1 \\
\hline$J(1.10-1.34 \mu \mathrm{m})$ & 2300 & 4.0 & 2200 & 3.5 & 1600 & 3.5 & 2300 & 4.9 & $\geq 2400^{b}$ & 4.0 & 2 \\
\hline$H K(1.46-2.46 \mu \mathrm{m})$ & 1800 & 5.0 & 2000 & 3.0 & 1800 & 5.0 & 1850 & 5.0 & 1600 & 4.5 & 1 \\
\hline \multicolumn{12}{|c|}{ AB Pic B } \\
\hline$J H K(1.10-2.46 \mu \mathrm{m})$ & 1700 & 5.0 & 1600 & 3.5 & 1600 & 4.5 & 1800 & 4.8 & 1400 & 4.5 & 1 \\
\hline$J(1.10-1.34 \mu \mathrm{m})$ & 2400 & 4.5 & 2300 & 4.0 & 1600 & 3.5 & 2400 & 4.9 & $\geq 2400^{b}$ & 4.0 & 2 \\
\hline$H K(1.46-2.46 \mu \mathrm{m})$ & 2500 & 3.5 & 2300 & 3.0 & 1800 & 5.0 & 2300 & 5.0 & 1700 & 5.0 & 1 \\
\hline
\end{tabular}

Notes. ${ }^{(a)}$ These star formation region members have been dereddened as described in Sect. $4 .{ }^{(b)}$ Note that this $T_{\text {eff }}$ falls at the edge of the grid used.

in (Allers et al. 2007) and shown to be sensitive to surface gravity, but independent of spectral type. For the six targets for which the $J$-band data had sufficient sensitivity (2M1207A, TWA 5B, AB Pic B, GSC08047 B, and 2M0141), five had indices ranging from 0.93 to 1.08 , within the range associated with giant stars and young brown dwarfs in star-forming regions (Allers et al. 2007). Only $2 \mathrm{M} 0141$ has a higher value of 1.14 , though this target may be older than the rest of the sample. The inferred values of $T_{\text {eff }}$ from the restricted grid model fits to the data are used to estimate the range of possible temperatures consistent with the observations. Uncertainties based on comparisons of the five different synthetic atmospheres should be more representative of the true range of values than uncertainties estimated from a comparison with a single model set.

The SINFONI data and best-fit atmosphere model (restricted grid) from each of the five models is shown in Figs. 6-8 for the three age groups. The region of the spectrum most difficult to fit for the models is the peak and slope on the blue side of the $H$ band. The models often show a steeper slope and higher peak than the data. The peak of the $H$-band is also shaped by the dust cloud opacity, which is largely flat across the $H$-band. A high cloud opacity can flatten the spectrum over this wavelength range, and may explain the model discrepancy in the $H$-band shape. The portion of the data with the lowest signal-to-noise is the blue edge of the $J$-band, and improving this section of the data would enable more reliable fits, as some of the targets show a range of model best fits with significantly different slopes across the $J$-band. AB Pic B presents the most unusual shape, with a $J$-band notably lower flux than the $H$-band and no model is able to match the combination of flux levels and spectral shape as well as the other targets without adding other effects artificially. Unlike 2M1207 B, the inferred radius is not anomalously low. The inferred radii of all targets is discussed in Sect. 6.2.3.

The results of the model fits to the full and partial spectra of each target are given in Table 7. Although some of the effective temperatures based on fits to subsections of the data are different for the full and restricted grids, the $T_{\text {eff }}$ values for all targets using the full $J, H, K$ spectrum differ by $100 \mathrm{~K}$ or less with 
A\&A 540, A85 (2012)
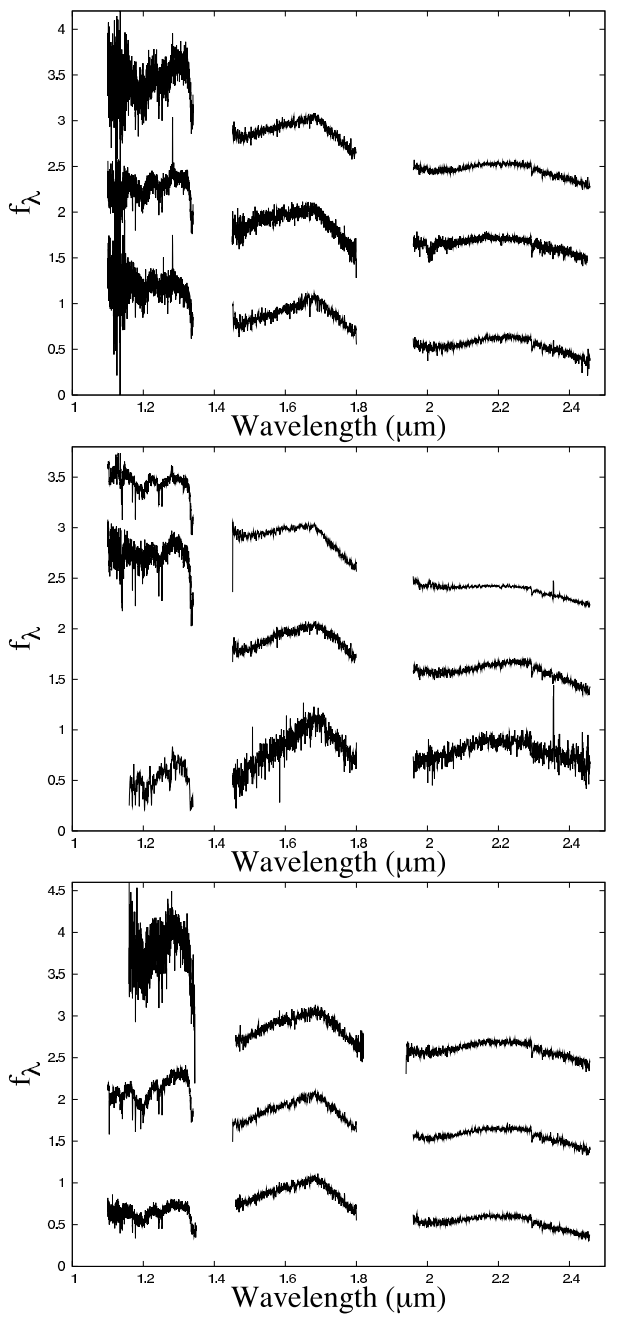

Fig. 5. SINFONI $J, H, K$ spectra of the sample, separated into three age groups: (top panel, top to bottom) the 1-3 Myr objects DH Tau B, GQ Lup B, and CT Cha B; (middle panel. top to bottom) the $8 \mathrm{Myr}$ objects 2M1207A, TWA 5B and 2M1207B; (bottom panel, top to bottom) the 30-50 Myr objects GSC 08047B, 2M0141, and AB Pic B. For each age group, the spectra are ordered from hottest (top) to coolest (bottom), and an offset has been added to the flux for ease of comparison. The spectra of the youngest subset have been dereddened based on the extinction measured for the primaries.

either the full grid or the restricted $\log (g)$ grid. The exception to this is $2 \mathrm{M} 1207 \mathrm{~B}$ where the difference in effective temperature is $200 \mathrm{~K}$. The trend of higher inferred temperatures from $J$-band fits compared to $H+K$-band fits is seen in both the full grid fits and the restricted $\log (g)$ fits, as shown in Fig. 9. The higher values of $T_{\text {eff }}$ from the $J$-band fits compared to the $H+K$ fits are most pronounced for the lower temperature objects below $2200 \mathrm{~K}$. There may be too much dust in the models in this temperature regime, resulting in underestimated $J$-band flux which a $J$-band fit compensates for with a bias towards higher $T_{\text {eff }}$ values.

Figure 10 shows the inferred $T_{\text {eff }}$ from each model as a function of the average $T_{\text {eff }}$ value. The calculation of the average excluded any values that corresponded to the limits of a model grid, and those limits are noted in Fig. 10. The range in best-fit temperatures for a given object is $\pm 150-300 \mathrm{~K}$, considering all of the five different models. While no model always suggests the highest or lowest temperature, the DUSTY and Gaia-Dusty models give typically higher effective temperatures, and the Drift-PHOENIX and Marley et al. models are usually associated with the cooler temperature fits for each target, which is consistent with the typically lower amount of dust compared to the DUSTY models.

\subsubsection{Inferred radii}

The fitting procedure to obtain the best match for effective temperature from each model grid includes adjusting the object radius until the flux level corresponds to the synthetic spectrum. For the restricted $\log (g)$ fits reported in Table 7, each model $T_{\text {eff }}$ and $\log (g)$ combination has an associated object radius. The range of inferred radii from the five separate models is given in Table 8; values based on best-fits at an extreme of a model grid are excluded. Although the radius is estimated from a restricted $\log (g)$ atmosphere model grid, the radius is not fixed by the value of $\log (g)$.

As expected for young objects in the process of contracting, the radii are larger than the $\sim 0.1 R_{\odot}$ predicted for older field planets and brown dwarfs (Baraffe et al. 2003). The only exception is $2 \mathrm{M} 1207 \mathrm{~B}$, and the apparent small radius - or underluminosity - of this object has been reassessed with updated models different from those considered in this study and found to be have an inferred radius consistent with evolutionary models (Barman et al. 2011). Due to the young ages of the targets, the radii may retain an imprint from the initial conditions, including the early accretion history of the objects (e.g., Baraffe et al. 2002; Fortney et al. 2008; Baraffe \& Chabrier 2010). Figure 11 plots the inferred radii of the targets as a function of age, along with the evolutionary tracks for the size of 5-30 $M_{\text {Jup }}$ objects. The data exhibit a systematic decline with stellar age and are largely within the range predicted by the models (Baraffe et al. 2003).

\subsubsection{Metallicity grid}

For one of the model grids - the Drift-PHOENIX set (Witte et al. 2009) - a range of non-solar metallicities are also included, and the targets were fit with the expanded library of synthetic spectra to investigate the impact of metallicity on the inferred physical properties of $T_{\text {eff }}$ and $\log (g)$. The results of the metallicity comparison are given in Table 9, including fits to both the full spectrum and the $J$ - and $H+K$-bands. For approximately half the targets, the best fit metallicity over the full spectrum was the solar value, supporting the use of solar metallicities for these young stars. For the remaining targets with best fit non-solar metallicities, the impact on the effective temperature and surface gravity estimates was minimal and resulted in a change of one step size in $\log (g)$ or $T_{\text {eff }}$, except in one case. For DH Tau B, the difference in best fit effective temperatures is $700 \mathrm{~K}$. Overall, the ability to fit for metallicity does not typically change the inferred properties by an amount larger than the uncertainty estimated from the range in values from the different models.

\subsection{Comparison with previous observations}

\subsubsection{Effective temperatures}

The inferred effective temperatures for the majority of the sample are consistent with previous estimates, however two of the targets - CT Cha B and AB Pic B - are notably different. In both cases, the range of best fit values for $T_{\mathrm{eff}}$ is shifted to cooler temperatures, with the range of $T_{\text {eff }} 1500-1950 \mathrm{~K}$ for CT Cha B compared to 2350-2850 K from previous estimates (Schmidt et al. 2008) and 1400-1800 K for AB Pic B compared 
J. Patience et al.: Near-IR spectra across the brown dwarf/planet boundary
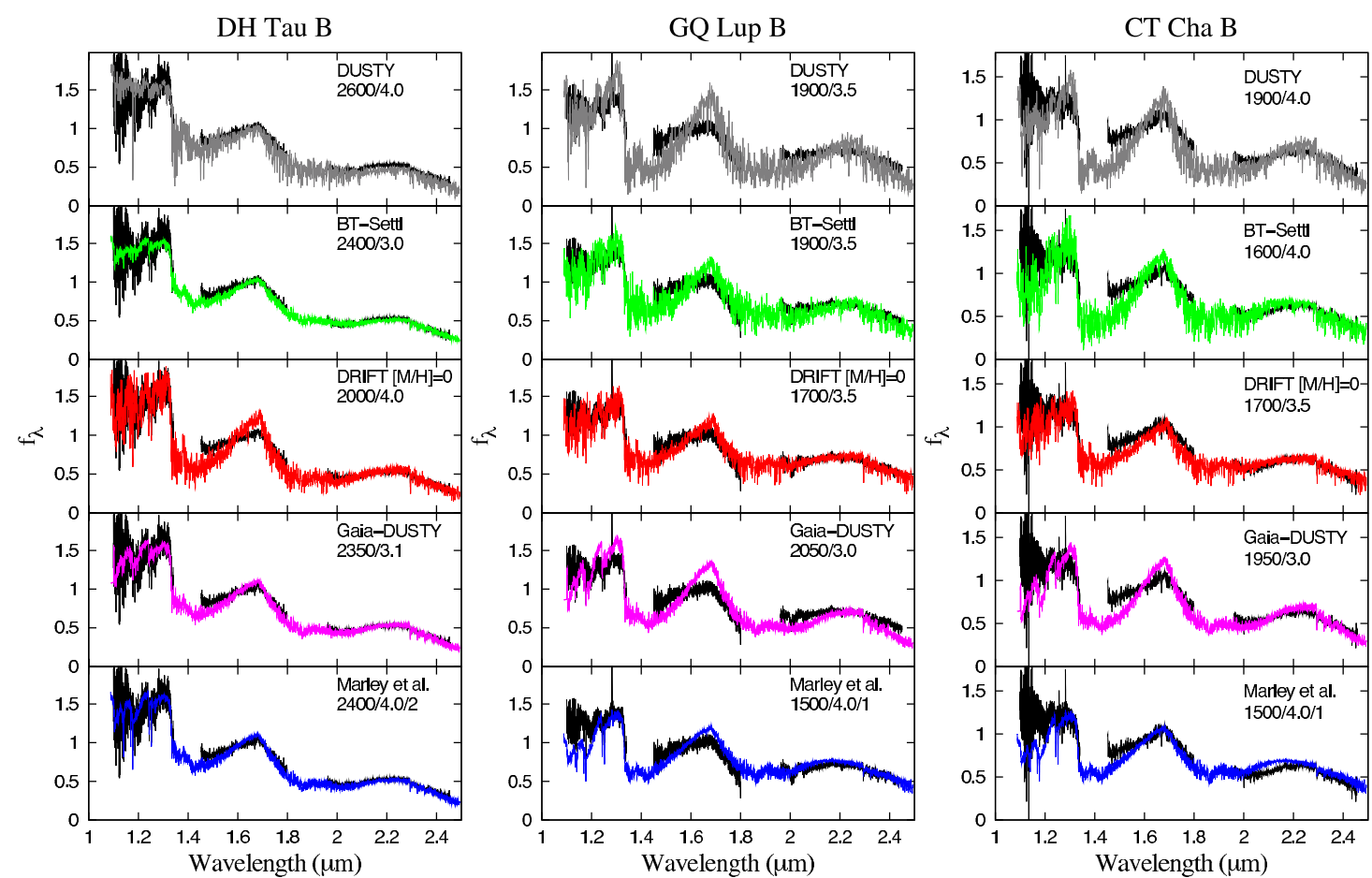

Fig. 6. The SINFONI spectra (black lines) and best-fit model (colored lines) to the full spectrum is plotted for the star-forming region targets: DH Tau B (left), GQ Lup B (middle), and CT Cha B (right). The restricted $\log (g)$ model grids are used.
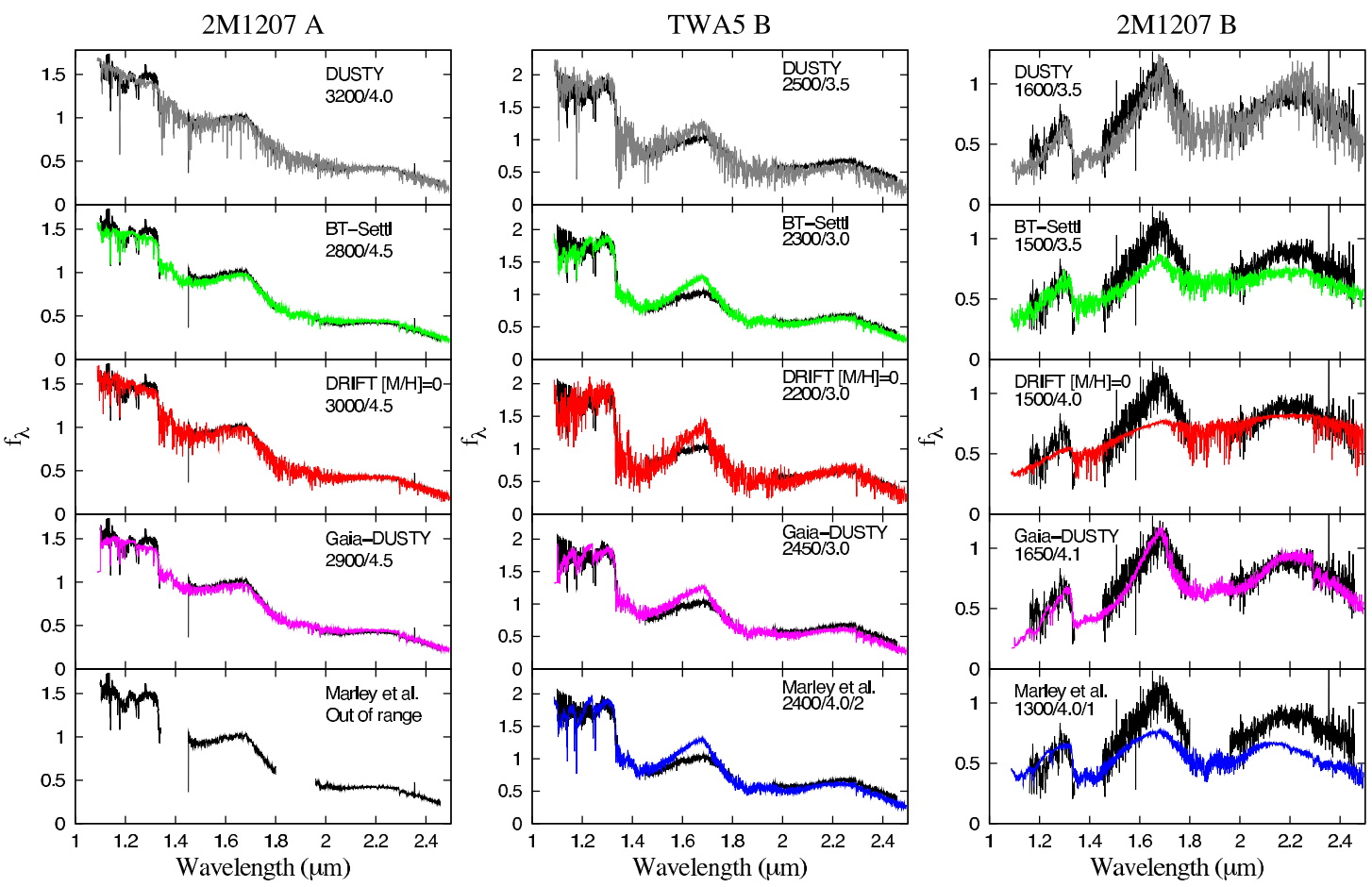

Fig. 7. The SINFONI spectra (black lines) and best-fit model (colored lines) to the full spectrum is plotted for the TW Hydra member targets: 2M1207 A (left), TWA 5B (middle), and 2M1207 B (right). The restricted $\log (g)$ model grids are used.

to $1700-2100 \mathrm{~K}$ from the literature (Bonnefoy et al. 2010). The implications for the masses implied from evolutionary models (Chabrier et al. 2000) are shown in Fig. 12 for CT Cha B and in Fig. 13 for AB Pic B. The lower effective temperatures are associated with lower masses, overlapping with the planetary mass regime.

The origin of the discrepancy in inferred $T_{\text {eff }}$ for CT Cha B is clear - the different treatment of extinction. By treating $A_{V}$ as a free parameter rather than using the value measured for the primary, Schmidt et al. (2008) find a much higher extinction which results in a large change in slope across the $J-K$ range. For AB Pic B, a previous model comparison (Bonnefoy et al. 2010) considered each of the $J, H$ and $K$ bands separately, and so the different results reported here arise from the systematically higher temperatures derived from fits restricted to the $J$-band, as shown in Fig. 6. The $T_{\text {eff }}$ values in Table 7 estimated from the $J$-band 

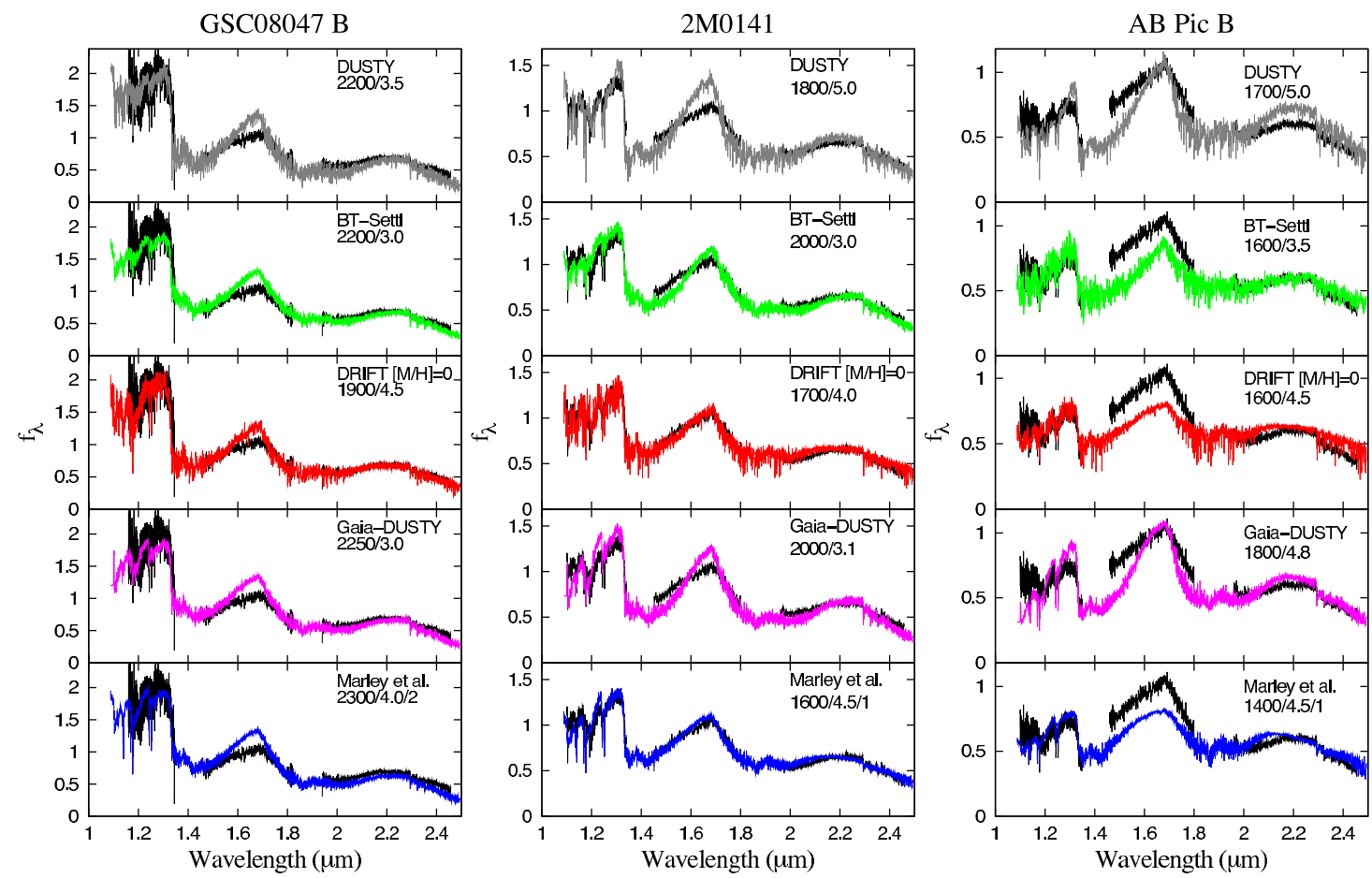

Fig. 8. The SINFONI spectra (black lines) and best-fit model (colored lines) to the full spectrum is plotted for the Tucana-Horologium and Field targets: GSC 08047 B (left), 2M0141 (middle), and AB Pic B (right). The restricted log $(g)$ model grids are used.
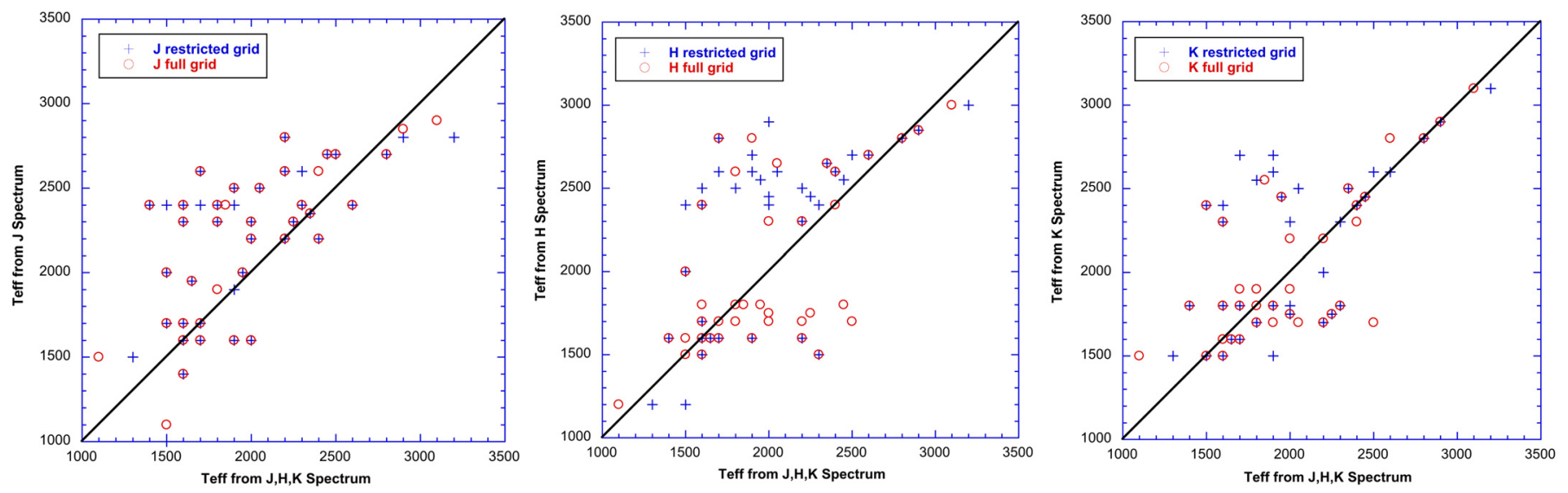

Fig. 9. The best-fit effective temperature inferred from a single bandpass (left: $J$-band, middle: $H$-band, right: $K$-band) portion of the spectrum of each companion as a function of the effective temperature based on the full $J, H, K$ spectrum. Results from the full grid are shown with open circles and from the restricted $\log (g)$ grid with crosses. All five models give systematically higher temperatures from fits using only the $J$-band spectrum.

portion of the spectrum are comparable to the upper bound of the previously reported effective temperature. In both cases, obtaining spectra over a larger wavelength range will enable more accurate estimates of $T_{\text {eff }}$ and determine if CT Cha B and AB Pic $\mathrm{B}$ are analogues of 2M1207 B and represent wide orbit companions with planetary masses, ideal cases for high signal-to-noise measurements of very low mass companions.

\subsubsection{Variability}

For two of the targets, DH Tau B and 2M0141, there are previously reported $J, H, K$ spectra taken at different epochs from the SINFONI data, and the combination of data sets are used to test for variability in the spectral shape, such as observed for the young low mass object TWA 30 in the TW Hydra association
(Looper et al. 2010). DH Tau B and 2M0141 are also young objects bracketing the age of TWA 30, making these targets interesting comparisons to TWA 30. The first epoch of DH Tau B spectra were obtained in 2003 ( $K$-band) and $2004(J+H$-band) with CISCO at Subaru (Itoh et al. 2005), several years prior to the SINFONI observations in 2007 (Table 3). Over the 3-4 year time period between observations, the spectrum shape does not change. The first epoch of the $2 \mathrm{M} 0141$ observations were obtained in 2004 with Spex on the IRTF (Kirkpatrick et al. 2006), two years prior to the 2006 SINFONI observations (Table 3). Like DH Tau B, no variability in the spectral shape is seen. It is not possible to search for a grey brightening or dimming of the spectrum, such as that reported in Apai et al. (2012, submitted) because the flux calibration of the SINFONI data relies on the same photometry used to calibrate the first epoch 


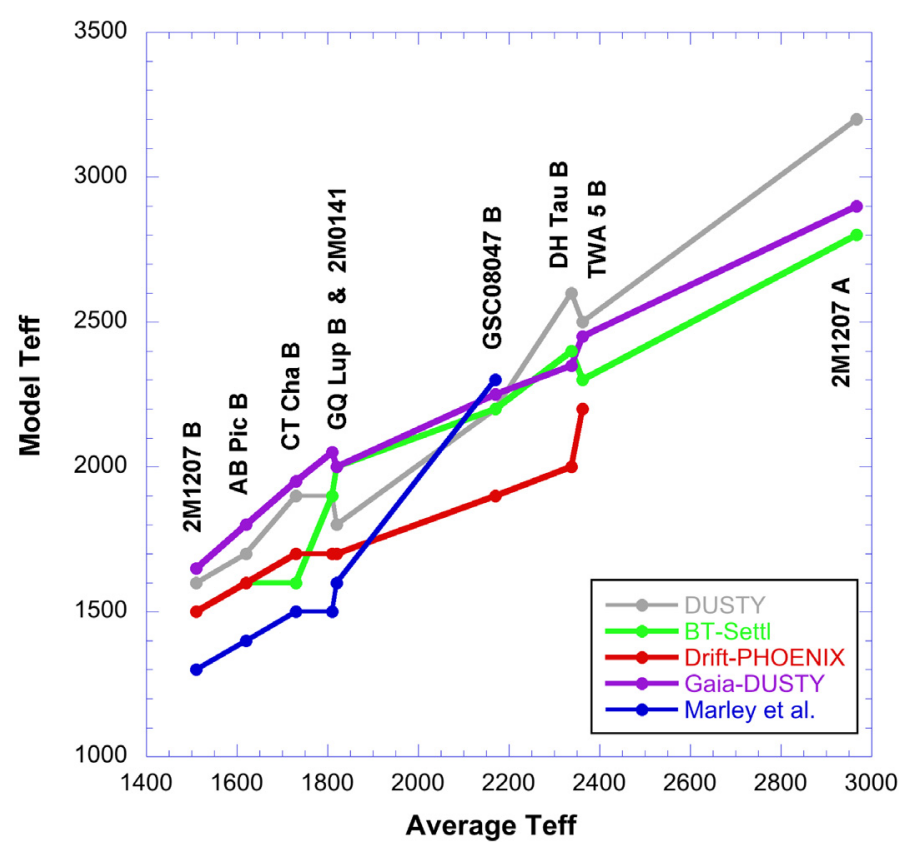

Fig. 10. The best-fit effective temperature of each object inferred from the restricted $\log (g)$ fits to each model (DUSTY - grey, BT-Settl green, Drift-PHOENIX - red, Gaia-Dusty - purple, Marley et al. - blue) plotted against the average of all the plotted model fits, excluding values at the edge of each grid. Considering all models, each object has a range of $\pm 150-300 \mathrm{~K}$, and the dispersion in temperature does not show a clear trend as a function of average effective temperature.

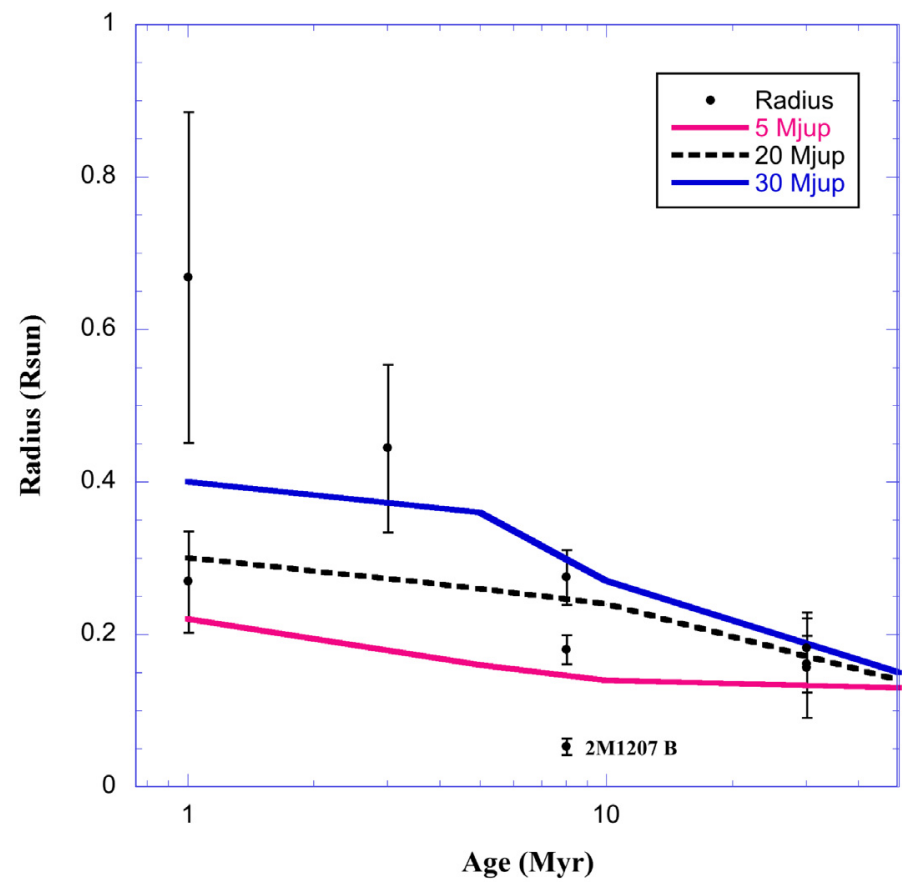

Fig. 11. The radius as a function of age for the targets (filled circles) and evolutionary tracks (Baraffe et al. 2003) for several substellar objects: $5 M_{\text {Jup }}$ (pink line), $20 M_{\text {Jup }}$ (black dashed line), and $30 M_{\text {Jup }}$ (blue line).

spectra,. Additional coordinated photometric and spectroscopic measurements are required to determine the frequency, wavelength dependence and physical origin of variability in low mass object atmospheres.
Table 8. Inferred radii.

\begin{tabular}{lc}
\hline \hline Target & Radius $\left(R_{\odot}\right)$ \\
\hline \multicolumn{2}{c}{$1-3 \mathrm{Myr}$} \\
DH Tau B & $0.20-0.34$ \\
GQ Lup B & $0.45-0.89$ \\
CT Cha B & $0.34-0.55$ \\
\hline \multicolumn{2}{c}{$8 \mathrm{Myr}$} \\
2M1207 A & $0.16-0.20$ \\
TWA 5B & $0.24-0.31$ \\
2M1207 B & $0.043-0.065$ \\
\hline \multicolumn{2}{c}{$30-50 \mathrm{Myr}$} \\
GSC 08047 B & $0.12-0.19$ \\
2MASS 0141 & $0.13-0.20$ \\
AB Pic B & $0.14-0.23$ \\
\hline
\end{tabular}

Table 9. Best fitting atmosphere model parameters, metallicity comparison.

\begin{tabular}{|c|c|c|c|c|c|}
\hline \multirow[t]{2}{*}{ Wavelength range } & \multicolumn{3}{|c|}{ Drift } & \multicolumn{2}{|c|}{ Drift, $[\mathrm{M} / \mathrm{H}]=0.0$} \\
\hline & $T_{\mathrm{eff}}(\mathrm{K})$ & $\log g$ & {$[\mathrm{M} / \mathrm{H}]$} & $T_{\text {eff }}(\mathrm{K})$ & $\log g$ \\
\hline \multicolumn{6}{|c|}{$\mathrm{DH} \mathrm{Tau} \mathrm{B}^{a}$} \\
\hline$J H K(1.10-2.46 \mu \mathrm{m})$ & 2700 & 3.5 & -0.6 & 2000 & 4.0 \\
\hline$J(1.10-1.34 \mu \mathrm{m})$ & 1600 & 3.5 & 0.0 & 1600 & 3.5 \\
\hline$H K(1.46-2.46 \mu \mathrm{m})$ & 2700 & 3.0 & -0.6 & 2700 & 4.0 \\
\hline \multicolumn{6}{|c|}{ GQ Lup B ${ }^{a}$} \\
\hline$J H K(1.10-2.46 \mu \mathrm{m})$ & 1700 & 3.5 & 0.0 & 1700 & 3.5 \\
\hline$J(1.10-1.34 \mu \mathrm{m})$ & 2600 & 3.5 & -0.6 & 2600 & 4.0 \\
\hline$H K(1.46-2.46 \mu \mathrm{m})$ & 1600 & 4.0 & -0.6 & 1700 & 4.0 \\
\hline \multicolumn{6}{|c|}{ CT Cha B ${ }^{a}$} \\
\hline$J H K(1.10-2.46 \mu \mathrm{m})$ & 1600 & 3.0 & -0.6 & 1700 & 3.5 \\
\hline$J(1.10-1.34 \mu \mathrm{m})$ & 1700 & 3.0 & 0.0 & 1700 & 3.0 \\
\hline$H K(1.46-2.46 \mu \mathrm{m})$ & 1800 & 4.0 & 0.3 & 1800 & 4.0 \\
\hline \multicolumn{6}{|c|}{ 2MASS 1207 A } \\
\hline$J H K(1.10-2.46 \mu \mathrm{m})$ & 2900 & 4.5 & -0.6 & $3000^{b}$ & 4.5 \\
\hline$J(1.10-1.34 \mu \mathrm{m})$ & 2900 & 4.5 & -0.6 & $2900^{b}$ & 4.5 \\
\hline$H K(1.46-2.46 \mu \mathrm{m})$ & 2900 & 4.5 & -0.6 & $3000^{b}$ & 4.5 \\
\hline \multicolumn{6}{|c|}{ TWA 5 B } \\
\hline$J H K(1.10-2.46 \mu \mathrm{m})$ & 2200 & 3.0 & 0.0 & 2200 & 3.0 \\
\hline$J(1.10-1.34 \mu \mathrm{m})$ & 2700 & 4.0 & -0.6 & 2800 & 4.5 \\
\hline$H K(1.46-2.46 \mu \mathrm{m})$ & 1800 & 4.0 & 0.3 & 1700 & 4.5 \\
\hline \multicolumn{6}{|c|}{ 2MASS 1207 B } \\
\hline$J H K(1.10-2.46 \mu \mathrm{m})$ & 1700 & 4.0 & 0.3 & 1500 & 4.0 \\
\hline$J(1.10-1.34 \mu \mathrm{m})$ & 1500 & 3.5 & -0.6 & 1700 & 4.0 \\
\hline$H K(1.46-2.46 \mu \mathrm{m})$ & 1700 & 4.0 & 0.3 & 1500 & 3.0 \\
\hline \multicolumn{6}{|c|}{ GSC $08047 \mathrm{~B}$} \\
\hline$J H K(1.10-2.46 \mu \mathrm{m})$ & 1900 & 4.5 & 0.0 & 1900 & 4.5 \\
\hline$J(1.10-1.34 \mu \mathrm{m})$ & 1600 & 3.5 & 0.0 & 1600 & 3.5 \\
\hline$H K(1.46-2.46 \mu \mathrm{m})$ & 1800 & 4.5 & 0.3 & 1700 & 4.0 \\
\hline \multicolumn{6}{|c|}{ 2MASS 0141} \\
\hline$J H K(1.10-2.46 \mu \mathrm{m})$ & 1700 & 4.0 & 0.0 & 1700 & 4.0 \\
\hline$J(1.10-1.34 \mu \mathrm{m})$ & 1600 & 3.5 & 0.0 & 1600 & 3.5 \\
\hline$H K(1.46-2.46 \mu \mathrm{m})$ & 1800 & 5.0 & 0.3 & 1800 & 5.0 \\
\hline \multicolumn{6}{|c|}{ AB Pic B } \\
\hline$J H K(1.10-2.46 \mu \mathrm{m})$ & 1600 & 4.5 & 0.0 & 1600 & 4.5 \\
\hline$J(1.10-1.34 \mu \mathrm{m})$ & 1800 & 4.5 & 0.3 & 1600 & 3.5 \\
\hline$H K(1.46-2.46 \mu \mathrm{m})$ & 1800 & 5.0 & 0.0 & 1800 & 5.0 \\
\hline
\end{tabular}

Notes. ${ }^{(a)}$ These star formation region members have been dereddened as described in Sect. 4. ${ }^{(b)}$ Note that this $T_{\text {eff }}$ falls at the extremes of the grid used.

\section{Summary and conclusions}

With data from the SINFONI integral field unit, an empirical grid of $J, H, K$ spectra for young $\sim 1-50$ Myr substellar objects has been constructed and compared with a suite of five 


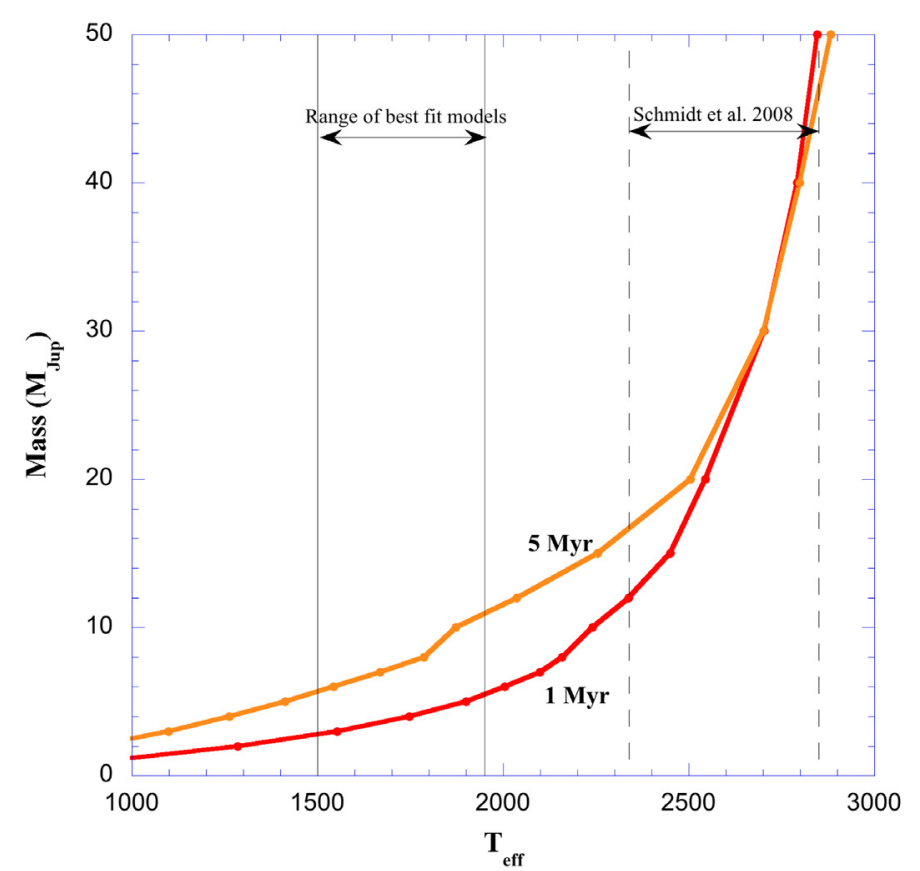

Fig. 12. The mass as a function of $T_{\text {eff }}$ for substellar objects, according to evolutionary models of $1 \mathrm{Myr}$ and $5 \mathrm{Myr}$ objects (Chabrier et al. 2000). The range of effective temperatures for CT Cha B from the best fit models is shown with solid lines and the range previously reported (Schmidt et al. 2008) is shown with dashed lines for comparison. The age of CT Cha B is expected to be between $1 \mathrm{Myr}$ and $5 \mathrm{Myr}$.

theoretical atmospheric model grids - DUSTY (Allard et al. 2001), BT-Settl (Allard et al. 2003, 2010), Drift-PHOENIX (Helling et al. 2008b; Witte et al. 2009), Gaia-Dusty (Rice et al. 2010), and Marley et al. (Ackerman \& Marley 2001; Marley et al. 2003; Saumon \& Marley 2008; Freedman et al. 2008). The spectra should also serve as observational comparisons for updated models including more advanced treatment of physical processes. Since most of these objects are companions, there is additional information on the age and extinction of the substellar object to aid in the model fitting. All spectra show the distinct triangular shape over the $H$-band, a region of the spectrum which was found to be particularly difficult for most models to match in detail.

Based on the best-fit synthetic atmosphere spectrum for each target from each restricted $\log (g)$ model grid, the uncertainty in the temperature estimate for a young, substellar object is \pm 150 $300 \mathrm{~K}$ from fits to the $J, H, K$ spectrum. This is considerably larger than the uncertainty usually inferred from comparisons to a single model grid ( $\sim 50-100 \mathrm{~K}$, cf. Burrows et al. 2006). For all targets except $2 \mathrm{M} 1207 \mathrm{~B}$, the inferred radius from the fit is greater than $0.1 R_{\odot}$, and generally consistent with the expectations of evolutionary models of young brown dwarfs (Baraffe et al. 2003).

We note that fits to small wavelength ranges do not account for the broad spectral morphology and so may suggest effective temperatures incompatible with observations of a broader range in wavelength. Fitting only the $J$-band portion results in systematically higher temperatures than fitting the $H+K$-band, likely due to systematic uncertainties in the model dust opacities. When the spectrum covers the full $J, H, K$ range, the inferred temperatures are approximately the same whether or not the model grids include surface gravities limited to the range for young objects or expanded to include the higher values of older objects. While the temperatures were consistent using the full

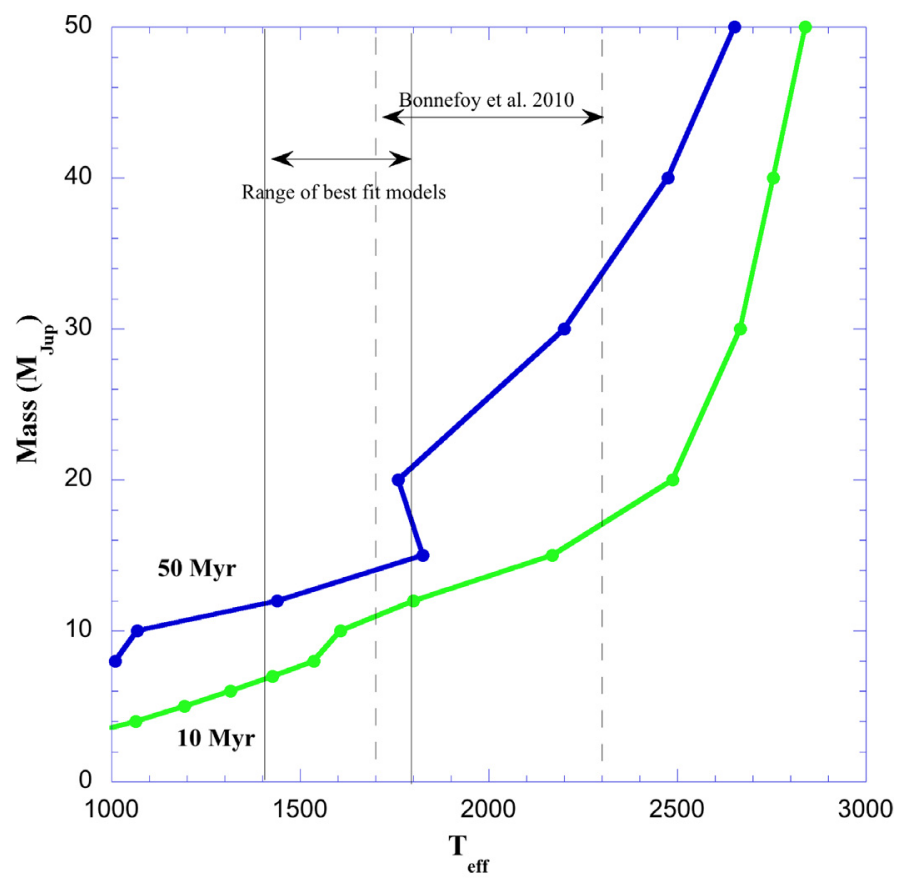

Fig. 13. The mass as a function of $T_{\text {eff }}$ for substellar objects, according to evolutionary models of $10 \mathrm{Myr}$ and $50 \mathrm{Myr}$ objects (Chabrier et al. 2000). The range of effective temperatures for AB Pic B from the best fit models is shown with solid lines and the range previously reported (Bonnefoy et al. 2010) is shown with dashed lines for comparison. The age of AB Pic B is expected to be between $10 \mathrm{Myr}$ and $50 \mathrm{Myr}$.

or restricted $\log (g)$ model grids, it is important to note that the spectral shape fit to the full model grid did not find the expected low surface gravities of these objects with known young ages. This apparent inconsistency is due to the fact that differences in spectral morphology caused by different effective temperatures dominate over spectral changes due to low surface gravity. Empirical comparison with observed spectra such as these may provide a more reliable method to determine the surface gravity; specific spectral lines and indices will be investigated in a follow-up paper (King et al., in prep.). For the Drift-PHOENIX model grid, it was possible to include fits to non-solar metallicities, and varying the metallicity did not impact the inferred temperatures by more than the uncertainty estimated from the range of model fits presented here.

From comparison of the SINFONI data with previous spectra of two targets, there is no evidence of substantial changes in the spectral shape, however more observations and coordinated photometry and spectroscopy are required to rule out variability in these young substellar objects. The spectrum of AB Pic B shows an unusually low $J$-band spectrum relative to the $H$-band spectrum, analogous to $2 \mathrm{M} 1207 \mathrm{~B}$, and the overall shape of the spectrum is more difficult to fit with the model grid than the remaining targets. For CT Cha B and AB Pic B, the new fits to the spectra suggest that the objects may have lower temperatures and, consequently, lower masses compared to previous estimates (Schmidt et al. 2008; Bonnefoy et al. 2010). Additional wavelength coverage in the spectra will refine the temperature estimates to determine if these companions represent additional examples of planetary mass companions that can be investigated with high signal-to-noise spectroscopy, like 2M1207 B.

Acknowledgements. We gratefully acknowledge grant support to Exeter from the Leverhulme Trust $(\mathrm{F} / 00144 / \mathrm{BJ})$ for J.P. and R.R.K., and STFC (ST/F003277/1) for J.P. and A.V. The studentship for R.D.R. is provided by 
STFC (ST/F007124/1). Ch.H. acknowledges an ERC starting grant from the the EU program FP7 Ideas for the LEAP project. We thank F. Allard, T. Barman, M. Marley, and D. Saumon for constructive comments and providing theoretical model grids used in this analysis, and we thank the referee for suggestions that improved the paper.

\section{References}

Ackerman, A. S., \& Marley, M. S. 2001, ApJ, 556, 872

Allard, F., Hauschildt, P. H., Alexander, D. R., Tamanai, A., \& Schweitzer, A. 2001, ApJ, 556, 357

Allard, F., Guillot, T., Ludwig, H.-G., et al. 2003, in Brown Dwarfs, ed. E. Martín (San Francisco: ASP), Proc. IAU Symp., 211, 325

Allard, F., Homeier, D., \& Freytag, B. 2010 [arXiv: 1011. 5405]

Allers, K. N., Jaffe, D. T., Luhman, K. L., et al. 2007, ApJ, 657, 511

Baraffe, I., \& Chabrier, G. 2010, A\&A, 521, 44

Baraffe, I., Chabrier, G., Allard, F., \& Hauschildt, P. H. 2002, A\&A, 382, 563

Baraffe, I., Chabrier, G., Barman, T. S., Allard, F., \& Hauschildt, P. H. 2003, A\&A, 402, 701

Barman, T. S., Macintosh, B., Konopacky, Q. M., \& Marois, C. 2011, ApJ, 735, L39

Batalha, C., Lopes, D. F., \& Batalha, N. M. 2001, ApJ, 548, 377

Becklin, E. E., \& Zuckerman, B. 1988, Nature, 336, 656

Bonnefoy, M., Chauvin, G., Rojo, P., et al. 2010, A\&A, 512, A52

Bonnet, H., Abuter, R., Baker, A., et al. 2004, The Messenger, 117, 17

Brandner, W., \& Zinnecker, H. 1997, A\&A, 321, 220

Burrows, A., Marley, M., Hubbard, W. B., et al. 1997, ApJ, 491, 856

Burrows, A., Sudarsky, D., \& Hubeny, I. 2006, ApJ, 640, 1063

Cardelli, J. A., Clayton, G. C., \& Mathis, J. S. 1989, ApJ, 345, 245

Carpenter, J. M. 2001, AJ, 121, 2851

Chabrier, G., Baraffe, I., Allard, F., \& Hauschildt, P. 2000, ApJ, 542, 464

Chauvin, G., Lagrange, A.-M., Dumas, C., et al. 2004, A\&A, 425, L29

Chauvin, G., Lagrange, A.-M., Dumas, C., et al. 2005a, A\&A, 438, L25

Chauvin, G., Lagrange, A.-M., Lacombe, F., et al. 2005b, A\&A, 430, 1027

Chauvin, G., Lagrange, A.-M., Zuckerman, B., et al. 2005c, A\&A, 438, L29

Cushing, M. C., Marley, M. S., Saumon, D., et al. 2008, ApJ, 678, 1372

Dumas, C., Kaufer, A., \& Hainaut, O. 2007, SINFONI data reduction cookbook VLT-AMN-ESO-14700-4037

Eisenhauer, F., Abuter, R., Bickert, K., et al. 2003, in Instrument Design and Performance for Optical/Infrared Ground-based Telescopes, Proc. SPIE, 4841, 1548

Fortney, J. J., Marley, M. S., Saumon, D., \& Lodders, K. 2008, ApJ, 683, 1104

Franco, G. A. P. 2002, MNRAS, 331, 474

Freedman, R. S., Marley, M. S., \& Lodders, K. 2008, ApJS, 174, 504

Gebbinck, M. K., Bellester, P., \& Peron, M. 2007, VLT Gasgano User Manual VLT-PRO-ESO-19000-1932

Gizis, J. E. 2002, ApJ, 575, 484

Hauschildt, P. H., Allard, F., \& Baron, E. 1999, ApJ, 512, 377

Hayes, D. S. 1985, Proc. of Calibration of fundamental stellar quantities, 111, 225

Helling, C., Ackerman, A., Allard, F., et al. 2008a, MNRAS, 391, 1854

Helling, C., Dehn, M., Woitke, P., \& Hauschildt, P. H. 2008b, ApJ, 675, L105
Ireland, M. J., Kraus, A., Martinache, F., Law, N., \& Hillenbrand, L. A. 2011, ApJ, 726, 113

Itoh, Y., Hayashi, M., Tamura, M., et al. 2005, ApJ, 620, 984

Itoh, Y., Tamura, M., Hayashi, M., et al. 2008, PASJ, 60, 209

Kirkpatrick, J. D., Barman, T. S., Burgasser, A. J., et al. 2006, ApJ, 639, 1120

Kleinmann, S. G., \& Hall, D. N. B. 1986, ApJS, 62, 501

Konopacky, Q. M., Ghez, A. M., Duchêne, G., McCabe, C., \& Macintosh, B. A. 2007, AJ, 133, 2008

Lafrenière, D., Jayawardhana, R., \& van Kerkwijk, M. H. 2008, ApJ, 689, L153

Lavigne, J.-F., Doyon, R., Lafrenière, D., Marois, C., \& Barman, T. 2009, ApJ, 704, 1098

Lodieu, N., Hambly, N. C., Jameson, R. F., \& Hodgkin, S. T. 2008, MNRAS, 383, 1385

Looper, D. L., Mohanty, S., Bochanski, J. J., et al. 2010, ApJ, 714, 45

Lowrance, P. J., McCarthy, C., Becklin, E. E., et al. 1999, ApJ, 512, L69

Lucas, P. W., Roche, P. F., Allard, F., \& Hauschildt, P. H. 2001, MNRAS, 326, 695

Luhman, K. L. 2004, ApJ, 602, 816

Luhman, K. L., Wilson, J. C., Brandner, W., et al. 2006, ApJ, 649, 894

Marley, M. S., Ackerman, A. S., Burgasser, A. J., et al. 2003, Proc. Brown Dwarfs, 211, 333

Marois, C., Macintosh, B., \& Barman, T. 2007, ApJ, 654, L151

McCarthy, C., \& Zuckerman, B. 2004, AJ, 127, 2871

McElwain, M. W., Metchev, S. A., Larkin, J. E., et al. 2007, ApJ, 656, 505

McGovern, M. R., Kirkpatrick, J. D., McLean, I. S., et al. 2004, ApJ, 600, 1020

Metchev, S. A., \& Hillenbrand, L. A. 2006, ApJ, 651, 1166

Modigliani, A., Mirny, K., Bellester, P., \& Peron, M. 2009, SINFONI pipeline User Manual VLT-AMN-ESO-19500-3600

Mohanty, S., Jayawardhana, R., Huélamo, N., \& Mamajek, E. 2007, ApJ, 657, 1064

Mountain, C. M., Selby, M. J., Leggett, S. K., Blackwell, D. E., \& Petford, A. D. 1985, A\&A, 151, 399

Nakajima, T., Oppenheimer, B. R., Kulkarni, S. R., et al. 1995, Nature, 378, 463

Neuhäuser, R., \& Guenther, E. W. 2004, A\&A, 420, 647

Neuhäuser, R., Guenther, E. W., Petr, M. G., et al. 2000, A\&A, 360, L39

Neuhäuser, R., Guenther, E. W., Wuchterl, G., et al. 2005, A\&A, 435, L13

Neuhäuser, R., Schmidt, T. O. B., Seifahrt, A., et al. 2009, Proc. Cool Stars, 1094,844

Patience, J., King, R. R., de Rosa, R. J., \& Marois, C. 2010, A\&A, 517, 76

Perryman, M. A. C., Lindegren, L., Kovalevsky, J., et al. 1997, A\&A, 323, L49

Prato, L., Greene, T. P., \& Simon, M. 2003, ApJ, 584, 853

Rice, E. L., Barman, T., McLean, I. S., Prato, L., \& Kirkpatrick, J. D. 2010, ApJS, 186, 63

Rice, E. L., Faherty, J. K., Cruz, K., et al. 2011 [arXiv: 1101.4231]

Saumon, D., \& Marley, M. S. 2008, ApJ, 689, 1327

Schmidt, T. O. B., Neuhäuser, R., Seifahrt, A., et al. 2008, A\&A, 491, 311

Seifahrt, A., Neuhäuser, R., \& Hauschildt, P. H. 2007, A\&A, 463, 309

Skemer, A. J., Close, L. M., Szúcs, L., et al. 2011, ApJ, 732, 107

Thatte, N. A., Tecza, M., Eisenhauer, F., et al. 1998, Proc. SPIE, 3353, 704

Webb, R. A., Zuckerman, B., Platais, I., et al. 1999, ApJ, 512, L63

White, R. J., \& Ghez, A. M. 2001, ApJ, 556, 265

Witte, S., Helling, C., \& Hauschildt, P. H. 2009, A\&A, 506, 1367

Zuckerman, B., \& Song, I. 2009, A\&A, 493, 1149 